\title{
Assessing Total Nitrogen in Surface-Water Samples-Precision and Bias of Analytical and Computational Methods
}

Scientific Investigations Report 2012-5281 



\section{Assessing Total Nitrogen in Surface-Water Samples-Precision and Bias of Analytical and Computational Methods}

By David L. Rus, Charles J. Patton, David K. Mueller, and Charles G. Crawford

Scientific Investigations Report 2012-5281 


\title{
U.S. Department of the Interior \\ KEN SALAZAR, Secretary \\ U.S. Geological Survey \\ Marcia K. McNutt, Director
}

\author{
U.S. Geological Survey, Reston, Virginia: 2012
}

For more information on the USGS - the Federal source for science about the Earth, its natural and living resources, natural hazards, and the environment, visit http://www.usgs.gov or call 1-888-ASK-USGS.

For an overview of USGS information products, including maps, imagery, and publications, visit http://www.usgs.gov/pubprod

To order this and other USGS information products, visit http://store.usgs.gov

Any use of trade, firm, or product names is for descriptive purposes only and does not imply endorsement by the U.S. Government.

Although this information product, for the most part, is in the public domain, it also may contain copyrighted materials as noted in the text. Permission to reproduce copyrighted items must be secured from the copyright owner.

Suggested citation:

Rus, D.L., Patton, C.J., Mueller, D.K., and Crawford, C.G., 2012, Assessing total nitrogen in surface-water samplesPrecision and bias of analytical and computational methods: U.S. Geological Survey Scientific Investigations Report 2012-5281, 38 p. 


\section{Contents}

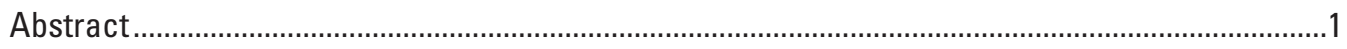

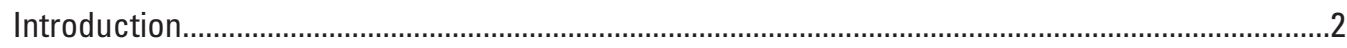

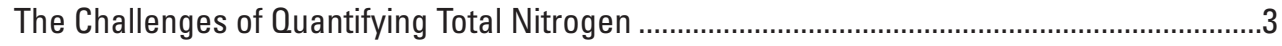

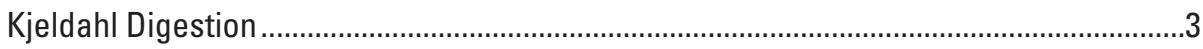

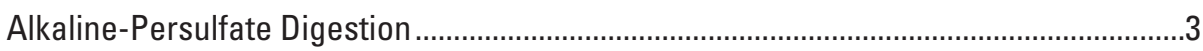

High-Temperature-Combustion Oxidation .................................................................

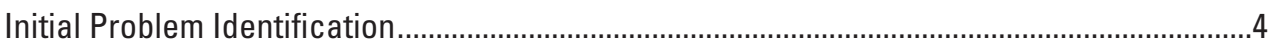

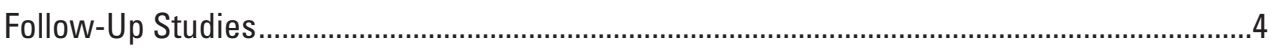

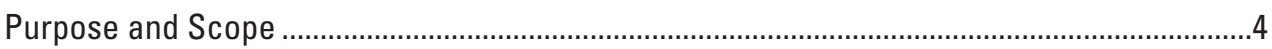

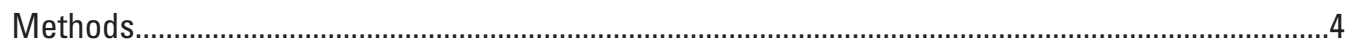

Laboratory-Experiment Design and Sample Analysis ......................................................

Synoptic-Field-Study Design and Sample Analysis ..................................................................

Dissolved-Nitrogen Procedures........................................................................................

Particulate-Nitrogen Procedures ...................................................................................

Selection of a Total-Nitrogen Reference for Evaluation of Bias in Synoptic-Field

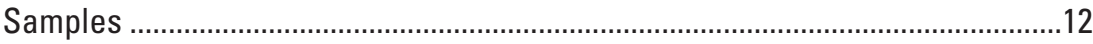

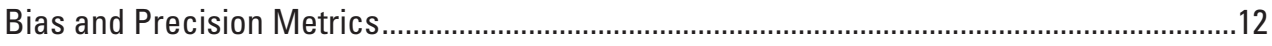

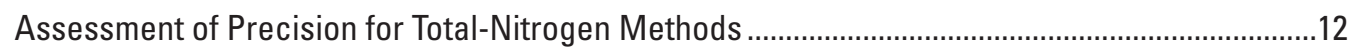

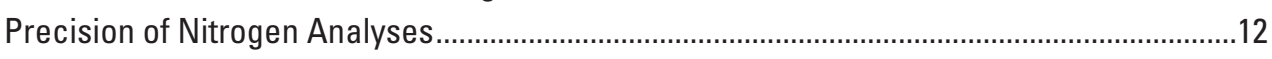

Factors That Could Affect the Variability of Particulate-Nitrogen Measurements .................14

Precision of Computed Total Nitrogen ...............................................................................

Assessment of Bias in Total-Nitrogen Methods.......................................................................17

Assessment of Bias Determined by the Laboratory Experiment .............................................17

Bias in the Alkaline-Persulfate and Kjeldahl Methods...................................................17

Source of Bias in the Alkaline-Persulfate Method ......................................................18

Source of Bias in the Kjeldahl-Digestion Method .......................................................20

Assessment of Bias Determined by the Synoptic-field Study.............................................21

Bias in the Alkaline-Persulfate-Digestion Method ..........................................................22

Spatial Patterns of Bias in Total Nitrogen Determined by Alkaline-Persulfate

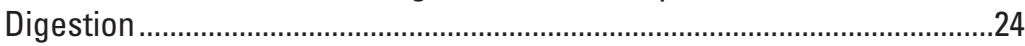

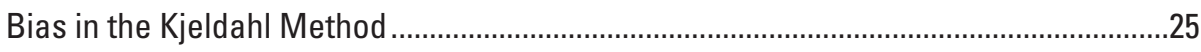

Evaluation of Alternatives for Determining Total Nitrogen ......................................................29

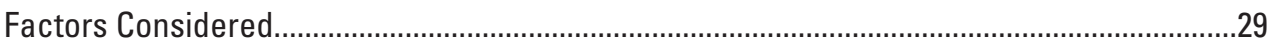

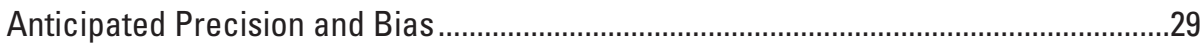

Impact on Field and Laboratory Procedures.................................................................31

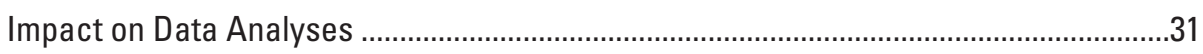

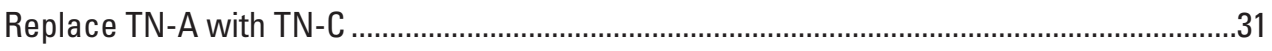

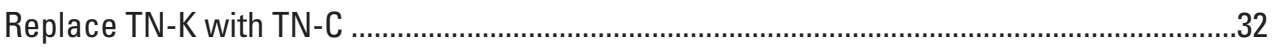

Add TN-C Analyses to Existing Analyses of TN-A or TN-K ………......................................32

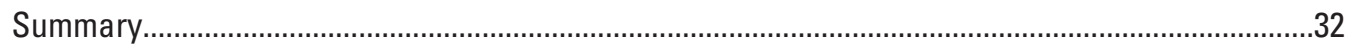

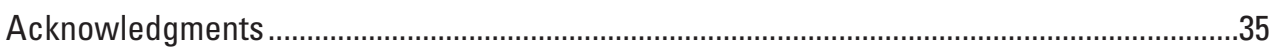

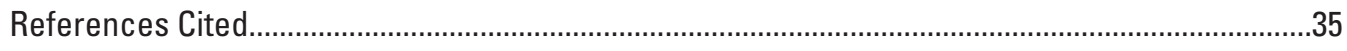

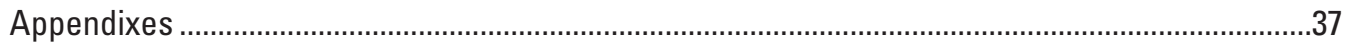

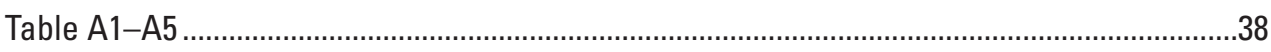




\section{Figures}

1. Graph showing nitrogen content measured for various amounts of three reference materials at the U.S. Geological Survey National Water Quality Laboratory, Denver, Colorado, in comparison to reference values determined at the U.S. Geological Survey Sediment Chemistry Laboratory, Atlanta, Georgia ...

2. Map showing distribution of sites sampled for the synoptic-field study of bias in total nitrogen by alkaline-persulfate digestion (TN-A) and as the sum of total Kjeldahl nitrogen and nitrate plus nitrite (TN-K), 2009-10

3. Graphs showing relations of standard deviation $(A, C, E$, and $G)$ and relative standard deviation $(B, D, F$, and $H)$ to mean concentration for selected nitrogen species in replicate samples collected for the synoptic-field study, 2009-10.

4. Graphs showing relations of $(A)$ suspended sediment concentration and $(B)$ water volume filtered to relative standard deviation of particulate-nitrogen measurements in replicate samples collected for the synoptic-field study, 2009-10.

5. Graph showing bias in total nitrogen determined by alkaline-persulfate digestion (TN-A) and total Kjeldahl nitrogen (TKN) in laboratory tests using synthetic samples made from nutrient-fortified water and various concentrations of two reference materials: Buffalo River sediment or Montana soil.

6. Graph showing comparison of bias in total-nitrogen concentration in synthetic samples analyzed using the standard alkaline-persulfate method and an alternate, fortified-reagent test using four times the reagent relative to the original sample volume

7. Graph showing apparent recovery of particulate nitrogen (assuming complete and accurate recovery of dissolved nitrogen species) in synthetic samples analyzed for total nitrogen by alkaline-persulfate digestion...

8. Graph showing apparent recovery of nitrate during Kjeldahl digestion (determined as the difference between measured and known concentrations of total Kjeldahl nitrogen in comparison to the known concentration of nitrate nitrogen) in synthetic samples amended with Buffalo River sediment or Montana soil.

9. Boxplots showing distribution of $(A)$ bias and $(B)$ relative bias in total nitrogen determined by alkaline-persulfate digestion (TN-A) and as the sum of total Kjeldahl nitrogen and nitrate plus nitrite (TN-K) observed in the synoptic-field study, 2009-10.

10. Graphs showing relations of bias in total nitrogen determined by alkaline-persulfate digestion (TN-A) in samples collected as part of the synoptic-field study to ( $A$ and $B$ ) suspended-sediment concentration and $(C)$ particulate-nitrogen concentration

11. Boxplots showing distribution of $(A)$ bias and $(B)$ relative bias in total nitrogen determined by alkaline-persulfate digestion (TN-A) in samples collected for the synoptic-field study and categorized by suspended-sediment concentration range ....24

12. Graphs showing general trends (as represented by locally weighted regression) of $(A)$ bias and $(B)$ relative bias in total nitrogen determined by alkaline-persulfate digestion (TN-A) in sediment and soil samples from the laboratory experiment and synoptic-field study .

13. Maps showing $(A)$ mean bias and $(B)$ mean relative bias in total nitrogen determined by alkaline-persulfate digestion (TN-A) for sampling sites with 10 or more measurements, synoptic-field study, 2009-10.

14. Boxplots showing comparisons of relative bias in total nitrogen determined by alkaline-persulfate digestion (TN-A) to drainage-basin characteristics for selected sampling sites in synoptic-field study, 2009-10, for $(A)$ percent silt and clay in topsoil, $(B)$ dominant land use, and $(C)$ dominant physiographic division 
15. Graphs showing relations of bias and relative bias in total nitrogen computed as the sum of total Kjeldahl nitrogen and nitrate plus nitrite (TN-K) to nitrate plus nitrite concentration in samples collected as part of the synoptic-field study, 2009-10..........28

16. Boxplots showing distribution of $(A)$ bias and $(B)$ relative bias in total nitrogen determined as the sum of total Kjeldahl nitrogen and nitrate plus nitrite (TN-K) observed in samples collected for the synoptic-field study, 2009-10, among ranges of nitrate plus nitrite concentration.

17. A graphical depiction of the relative effect of precision and bias on three methods for determining total nitrogen.....

\section{Tables}

1. Nitrogen analyses of samples collected from sites in the Mississippi River Basin during April and May, 2005.

2. Nitrogen content of reference materials determined by analyses at the U.S. Geological Survey Sediment Chemistry Laboratory, Atlanta, Georgia...

3. Nitrogen content of reference materials determined by analyses at the U.S. Geological Survey National Water Quality Laboratory in Denver, Colorado.

4. Sampling sites and number of samples collected for the synoptic-field study of bias in total nitrogen as determined by alkaline-persulfate digestion (TN-A) or the sum of total Kjeldahl nitrogen and nitrate plus nitrite (TN-K), 2009-10..

5. Estimates of variability in replicate measurements for nitrogen species.........................13

6. Summary of advantages and disadvantages associated with three alternatives to current U.S. Geological Survey practices for determining total-nitrogen concentration in surface-water samples.....

\section{Conversion Factors}

\begin{tabular}{|c|c|c|}
\hline Multiply & By & To obtain \\
\hline \multicolumn{3}{|c|}{ Length } \\
\hline millimeter (mm) & 0.03937 & inch (in.) \\
\hline micrometer $(\mu \mathrm{m})$ & 0.00003937 & inch (in.) \\
\hline \multicolumn{3}{|c|}{ Area } \\
\hline square kilometer $\left(\mathrm{km}^{2}\right)$ & 0.3861 & square mile $\left(\mathrm{mi}^{2}\right)$ \\
\hline \multicolumn{3}{|c|}{ Volume } \\
\hline liter (L) & 1.057 & quart (qt) \\
\hline milliliter $(\mathrm{mL})$ & 0.06102 & cubic inch $\left(\right.$ in $\left.^{3}\right)$ \\
\hline \multicolumn{3}{|c|}{ Mass } \\
\hline milligram (mg) & 0.00003527 & ounce, avoirdupois (oz) \\
\hline \multicolumn{3}{|c|}{ Pressure } \\
\hline kilopascal (kPa) & 0.1450 & $\begin{array}{l}\text { pound-force per square inch } \\
\left(\mathrm{lbf} / \mathrm{in}^{2}\right)\end{array}$ \\
\hline
\end{tabular}

Temperature in degrees Celsius $\left({ }^{\circ} \mathrm{C}\right)$ may be converted to degrees Fahrenheit $\left({ }^{\circ} \mathrm{F}\right)$ as follows: ${ }^{\circ} \mathrm{F}=\left(1.8 \times{ }^{\circ} \mathrm{C}\right)+32$

Concentrations of chemical constituents in water are given either in milligrams per liter (mg/L) or percent. 


\section{Glossary of Total-Nitrogen Methods}

DN dissolved nitrogen, measured by the use of alkaline-persulfate digestion of a filtered sample

$\mathrm{NO}_{x}$ dissolved nitrate $\left(\mathrm{NO}_{3}^{-}\right)$plus nitrite $\left(\mathrm{NO}_{2}^{-}\right)$, measured colorimetrically

PN particulate nitrogen, measured by high-temperature combustion oxidation of a filter pad

TN-A total nitrogen measured by the use of alkaline-persulfate digestion of a whole-water sample

TN-C total nitrogen, computed from DN + PN

$\mathrm{TN}-\mathrm{K}$ total nitrogen, computed from $\mathrm{TKN}+\mathrm{NO}_{\mathrm{x}}$

TKN total Kjeldahl nitrogen, measured by the use of Kjeldahl digestion of a whole-water sample. TKN represents the sum of ammonia, dissolved-organic nitrogen, and particulate nitrogen in the sample

\section{Abbreviations and Acronyms}

\begin{tabular}{|c|c|}
\hline BOS & U.S. Geological Survey Branch of Quality Systems \\
\hline DKN & dissolved Kjeldahl nitrogen \\
\hline DOC & dissolved organic carbon \\
\hline DON & dissolved organic nitrogen \\
\hline HTCO & high-temperature, combustion oxidation \\
\hline NASOAN & National Stream-Quality Assessment Network \\
\hline NAWOA & National Water-Quality Assessment Program \\
\hline NIST & National Institute of Standards and Technology \\
\hline NMN & National Monitoring Network \\
\hline NWQL & U.S. Geological Survey National Water Quality Laboratory, Lakewood, Colorado \\
\hline OW0 & U.S. Geological Survey Office of Water Quality, Reston, Virginia \\
\hline RSD & relative standard deviation \\
\hline TN & total nitrogen \\
\hline SCL & U.S. Geological Survey Sediment Chemistry Laboratory, Atlanta, Georgia \\
\hline SSC & suspended-sediment concentration \\
\hline USGS & U.S. Geological Survey \\
\hline
\end{tabular}




\title{
Assessing Total Nitrogen in Surface-Water Samples- Precision and Bias of Analytical and Computational Methods
}

\author{
By David L. Rus, Charles J. Patton, David K. Mueller, and Charles G. Crawford
}

\section{Abstract}

The characterization of total-nitrogen (TN) concentrations is an important component of many surface-waterquality programs. However, three widely used methods for the determination of total nitrogen-(1) derived from the alkaline-persulfate digestion of whole-water samples (TN-A); (2) calculated as the sum of total Kjeldahl nitrogen and dissolved nitrate plus nitrite (TN-K); and (3) calculated as the sum of dissolved nitrogen and particulate nitrogen (TN-C) all include inherent limitations. A digestion process is intended to convert multiple species of nitrogen that are present in the sample into one measureable species, but this process may introduce bias. TN-A results can be negatively biased in the presence of suspended sediment, and TN-K data can be positively biased in the presence of elevated nitrate because some nitrate is reduced to ammonia and is therefore counted twice in the computation of total nitrogen. Furthermore, TN-C may not be subject to bias but is comparatively imprecise. In this study, the effects of suspended-sediment and nitrate concentrations on the performance of these TN methods were assessed using synthetic samples developed in a laboratory as well as a series of stream samples. A 2007 laboratory experiment measured TN-A and TN-K in nutrient-fortified solutions that had been mixed with varying amounts of sediment-reference materials. This experiment identified a connection between suspended sediment and negative bias in TN-A and detected positive bias in TN-K in the presence of elevated nitrate. A 2009-10 synoptic-field study used samples from 77 stream-sampling sites to confirm that these biases were present in the field samples and evaluated the precision and bias of TN methods.

The precision of TN-C and TN-K depended on the precision and relative amounts of the TN-component species used in their respective TN computations. Particulate nitrogen had an average variability (as determined by the relative standard deviation) of 13 percent. However, because particulate nitrogen constituted only 14 percent, on average, of TN-C, the precision of the TN-C method approached that of the method for dissolved nitrogen (2.3 percent). On the other hand, total
Kjeldahl nitrogen (having a variability of 7.6 percent) constituted an average of 40 percent of TN-K, suggesting that the reduced precision of the Kjeldahl digestion may affect precision of the TN-K estimates. For most samples, the precision of TN computed as TN-C would be better (lower variability) than the precision of TN-K. In general, TN-A precision (having a variability of 2.1 percent) was superior to TN-C and TN-K methods.

The laboratory experiment indicated that negative bias in TN-A was present across the entire range of sediment concentration and increased as sediment concentration increased. This suggested that reagent limitation was not the predominant cause of observed bias in TN-A. Furthermore, analyses of particulate nitrogen present in digest residues provided an almost complete accounting for the nitrogen that was underestimated by alkaline-persulfate digestion. This experiment established that, for the reference materials at least, negative bias in TN-A was caused primarily by the sequestration of some particulate nitrogen that was refractory to the digestion process. TN-K biases varied between positive and negative values in the laboratory experiment. Positive bias in TN-K is likely the result of the unintended reduction of a small and variable amount of nitrate to ammonia during the Kjeldahl digestion process. Negative TN-K bias may be the result of the sequestration of a portion of particulate nitrogen during the digestion process.

Negative bias in TN-A was present across the entire range of suspended-sediment concentration (1 to 14,700 milligrams per liter $[\mathrm{mg} / \mathrm{L}])$ in the synoptic-field study, with relative bias being nearly as great at sediment concentrations below 10 $\mathrm{mg} / \mathrm{L}$ (median of -3.5 percent) as that observed at sediment concentrations up to $750 \mathrm{mg} / \mathrm{L}$ (median of -4.4 percent). This lent support to the laboratory-experiment finding that some particulate nitrogen is sequestered during the digestion process, and demonstrated that negative TN-A bias was present in samples with very low suspended-sediment concentrations. At sediment concentrations above $750 \mathrm{mg} / \mathrm{L}$, the negative TN-A bias became more likely and larger (median of -13.2 percent), suggesting a secondary mechanism of bias, such as reagent limitation. From a geospatial perspective, 
trends in TN-A bias were not explained by selected basin characteristics. Though variable, TN-K bias generally was positive in the synoptic-field study (median of 3.1 percent), probably as a result of the reduction of nitrate.

Three alternative approaches for assessing TN in surface water were evaluated for their impacts on existing and future sampling programs. Replacing TN-A with TN-C would remove the bias from subsequent data, but this approach also would introduce discontinuity in historical records. Replacing TN-K with TN-C would lead to the removal of positive bias in $\mathrm{TN}-\mathrm{K}$ in the presence of elevated nitrate. However, in addition to the issues that may arise from a discontinuity in the data record, this approach may not be applicable to regulatory programs that require the use of total Kjeldahl nitrogen for stream assessment. By adding TN-C to existing TN-A or TN-K analyses, historical-data continuity would be preserved and the transitional period could be used to minimize the impact of bias on data analyses. This approach, however, imposes the greatest burdens on field operations and in terms of analytical costs. The variation in these impacts on different sampling programs will challenge U.S. Geological Survey scientists attempting to establish uniform standards for TN sample collection and analytical determinations.

\section{Introduction}

Dissolved and particulate inorganic and organic nitrogen occurs in a variety of forms (species) in environmental waters. The diversity of nitrogen species and their distributions between dissolved and particulate phases complicate sample collection and laboratory analyses required to measure their concentrations, transport, and cycling in environmental aquatic systems. Additional complications arise because a generic water-quality indicator, such as total nitrogen, includes multiple species that might be determined at different levels of detection, accuracy, and precision. Digestion processes are often used to convert multiple, co-occurring species of nitrogen into one measureable species. If the digestion process is insufficient in converting all of the targeted species, a negative bias might result. If the digestion process converts nontargeted species, a positive bias might result. This report focuses on the challenges and limitations of three widely used methods for routine determination of total nitrogen in filtered-water and whole-water samples. Specifically, it explores how suspendedsediment and nitrate concentrations affect the performance of these methods, both in synthetic media - laboratory-formulated, nitrogen-fortified deionized-water suspensions of reference materials (river sediment and soil) - and in a set of more than 900 geographically and compositionally diverse water samples collected and processed at 77 U.S. Geological Survey (USGS) stream-sampling sites during June 2009 through September 2010.
Nitrogenous nutrients that collectively compose total nitrogen in environmental water include:

- Ammonium ion $\left(\mathrm{NH}_{4}^{+}\right)$and ammonia $\left(\mathrm{NH}_{3}\right)$,

- Nitrate $\left(\mathrm{NO}_{3}^{-}\right)$,

- Nitrite $\left(\mathrm{NO}_{2}^{-}\right)$,

- Dissolved organic nitrogen (DON), and

- Particulate nitrogen (PN).

Constituents of DON include urea, methylamines, amino acids, and proteins (Berman and Bronk, 2003; Pehlivanoglu and Sedlak, 2006; Maie and others, 2006). PN includes particulate organic nitrogen and any $\mathrm{NH}_{4}^{+}$that might be associated with mineral sediments.

A variety of nutrient-analysis methods are utilized by the USGS National Water Quality Laboratory (NWQL). In environmental waters, dissolved $\mathrm{NH}_{4}^{+}$and $\mathrm{NH}_{3}$ are in $\mathrm{pH}$-dependent equilibrium. Under ambient $\mathrm{pH}$ conditions (about 6.08.5 standard units), $\mathrm{NH}_{4}^{+}$predominates. Colorimetric methods are equally reactive with both species, which by convention are reported collectively as "ammonia" (Fishman, 1993). Nitrate is determined colorimetrically as nitrite after chemical reduction to that species (Fishman, 1993); therefore, these two species are reported collectively as $\mathrm{NO}_{2}^{-}+\mathrm{NO}_{3}^{-}$(henceforth referred to as $\mathrm{NO}_{\mathrm{X}}$ in this report). In principle, Kjeldahl digestion (Patton and Truitt, 2000; American Public Health Association, 2012) reduces organic and particulate nitrogen species, but not $\mathrm{NO}_{\mathrm{X}}$, to ammonia. Kjeldahl nitrogen is by definition, then, the sum of ammonia, DON, and PN. Determining ammonia in filtered-water and whole-water Kjeldahl digests, therefore, yields the operationally defined parameters of dissolved Kjeldahl nitrogen (DKN) and total Kjeldahl nitrogen (TKN), respectively. Until 2003, USGS monitoring programs typically estimated total nitrogen (TN) as the sum of TKN and wholewater $\mathrm{NO}_{\mathrm{x}}$. Since then, a method for direct determination of TN by an alkaline-persulfate digestion method (Patton and Kryskalla, 2003) has been available at the NWQL. Operationally, alkaline-persulfate digestion oxidizes all forms of nitrogen to nitrate. Therefore, determining nitrate in filtered-water and whole-water alkaline-persulfate digests provides a direct measure of dissolved nitrogen (DN) and TN, respectively. PN can be calculated as the difference between TN and DN or as the difference between TKN and DKN. Direct determination of PN also is possible by high-temperature, combustion oxidation (HTCO) elemental analysis (U.S. Environmental Protection Agency (USEPA) method 440.0, Zimmerman and others, 1997). Sample preparation for PN analysis occurs at collection sites and involves separating suspended sediment from a wellmixed, measured volume of whole water by vacuum or pressure filtration through a 25 -millimeter $(\mathrm{mm})$-diameter glassfiber filter with a nominal pore size of 0.7 micrometer $(\mu \mathrm{m})$. Summing PN and DN provides a third way to estimate TN. 
The three approaches for TN estimation described above are summarized in the Glossary in the front of this report as well as the in the list below:

1. TN-A is TN measured in alkaline-persulfate digests of whole-water samples,

2. $\mathrm{TN}-\mathrm{K}$ is $\mathrm{TKN}+\mathrm{NO}_{\mathrm{X}}$, and

3. $\mathrm{TN}-\mathrm{C}$ is $\mathrm{DN}+\mathrm{PN}$.

TN-K estimates of TN predominate in the USGS National Water Information System database (http://waterdata.usgs.gov/ nwis) and reports prior to 2003. Since then, direct determination of TN (TN-A) has become more common, particularly for samples collected for the USGS National Water-Quality Assessment (NAWQA) Program (http://water.usgs.gov/ nawqa/). Over the past few years, estimating $\mathrm{TN}$ by the TN-C approach has increased as a consequence of a growing awareness of apparent bias in TN-K and TN-A values.

\section{The Challenges of Quantifying Total Nitrogen}

These three approaches for determining or calculating total nitrogen are affected by field- and laboratory-sampling operations and by analytical limitations - some well-known and understood, others less so. TN data quality depends on the nontrivial exercise of collecting representative samples of environmental waters and the suspended sediment entrained in them. Especially important is maintaining a uniform distribution of suspended sediment when composited whole-water samples are split into various containers for shipment and storage prior to laboratory analyses. A USGS study by Capel and Larson (1996) documents the precision and accuracy resulting from use of cone and churn devices to split water samples.

\section{Kjeldahl Digestion}

In the USGS Kjeldahl method, water samples are digested at 370 degrees Celsius $\left({ }^{\circ} \mathrm{C}\right)$ with concentrated sulfuric acid containing potassium sulfate and catalytic amounts of mercuric sulfate (Patton and Truitt, 2000). Organic nitrogen compounds in sample digests are reduced to ammonia, which along with any ammonia originally present in samples prior to digestion, are measured by colorimetry. In practice, the Kjeldahl-nitrogen method has three deficiencies. First, its method detection limit for ammonia is higher than those of methods for other nitrogen species, which is problematic for studies in low-concentration nutrient regimes. During 1994-2000, NWQL reporting levels for DKN and TKN were 0.05 0.2 milligrams per liter $(\mathrm{mg} / \mathrm{L})$ as nitrogen $(\mathrm{N})$, whereas the reporting levels for dissolved ammonia and $\mathrm{NO}_{\mathrm{X}}$ were as low as 0.008 and $0.002 \mathrm{mg} / \mathrm{L}$ as N, respectively. Second, variability of the TKN analyte is high relative to other nitrogen species. In replicate samples collected for the NAWQA Program during 1992-2001, TKN variability was 7.6 percent, compared to 1.9 percent and 2.2 percent for dissolved ammonia and
$\mathrm{NO}_{\mathrm{x}}$, respectively (Mueller and Titus, 2005). Third, when samples contain substantial concentrations of nitrate, some fraction of it can be reduced to ammonia during Kjeldahl digestion (American Public Health Association, 2012; Patton and Truitt, 2000; Patton and Kryskalla, 2003, p. 23-25 and fig. 8). If this occurs, calculated concentrations of TN-K are positively biased because of some double counting of nitrate. Although well-known to crop and soil analysts (Pace and others, 1982; Wyland and Jackson, 1994), the potential for nitrate interference in Kjeldahl-nitrogen analysis is seldom addressed in environmental-water testing literature. Kjeldahl-nitrogen methods also pose safety, health, and waste-stream handling concerns to analysts and waste-disposal personnel. These analytical and operational drawbacks notwithstanding, USEPA National Pollutant Discharge Elimination System and Safe Drinking Water Act regulations (40 CFR Part 136.3) stipulate TKN rather than TN for wastewater and drinking-water assessment.

\section{Alkaline-Persulfate Digestion}

The NWQL developed an alkaline-persulfate digestion method for analysis of TN (Patton and Kryskalla, 2003) to achieve lower detection limits, produce more precise analytical results, improve sample-collection efficiency, and avoid the health and safety concerns associated with Kjeldahl-nitrogen methods. The USGS method was adapted from a widely applied method for analysis of TN in estuarine and marine waters (D'Elia and others, 1977). Additional references and discussion of analytical considerations for alkaline-persulfate digestion are available in the USGS method documentation (Patton and Kryskalla, 2003). Alkaline-persulfate digestion methods are attractive because, in principle, they provide a direct measure of DN and TN in filtered and whole-water samples. In this method, water samples are dosed with alkaline persulfate reagent, sealed, and heated for an hour at elevated temperature and pressure in an autoclave. During the digestion, all forms of nitrogen are oxidized to nitrate, which is then determined by colorimetry. A potential for negative bias when other oxidizable substances, such as organic carbon or certain particulate minerals, are present in samples had been noted for this method. The cause of this bias was hypothesized to be that the persulfate reagent might not be sufficient to oxidize all nitrogen species to nitrate. In order to assess persulfate reagent limitation by dissolved organic carbon (DOC), Patton and Kryskalla added increasing concentrations of glucose to deionized water containing ammonium at a concentration of $2.5 \mathrm{mg} / \mathrm{L}$ as $\mathrm{N}$. The onset of reagent limitation by DOC occurred at a carbon concentration of $150 \mathrm{mg} / \mathrm{L}$ (Patton and Kryskalla, 2003, p. 21-22), which is much greater than DOC concentrations normally found in environmental waters. Their study also explored the effects of nitrate on observed differences between TN-K and TN-A but did not consider suspended sediment in whole-water samples as a potential source of negative bias in the TN-A method. 


\section{High-Temperature-Combustion Oxidation}

In June 2000, the NWQL validated an HTCO method for routine, simultaneous determination of total particulate carbon and PN (Zimmerman and others, 1997; USGS Office of Water Quality [OWQ] Technical Memorandum 2000.08). In this method, glass-fiber filter pads used to collect suspended sediment from measured volumes of whole water are dried and then combusted at $980^{\circ} \mathrm{C}$ in a pure oxygen atmosphere. Nitrogen oxides produced from particulate nitrogen are subsequently reduced to molecular nitrogen and detected by thermal conductivity. Unlike the Kjeldahl and alkaline-persulfate methods, HTCO PN determinations do not require wet-chemical digestion and do not generate hazardous waste; however, filtering water with suspended-sediment concentrations (SSC) higher than about $1,000 \mathrm{mg} / \mathrm{L}$ at collection sites can be time consuming.

\section{Initial Problem Identification}

During April and May 2005, 10 large-volume (4080 liter [L]) water samples were collected at sites that were part of the USGS National Stream-Quality Accounting Network (NASQAN) (http://water.usgs.gov/nasqan/) in the Mississippi River Basin. These samples were sent to the USGS Sediment Chemistry Laboratory (SCL) in Atlanta, Ga., for processing and analysis. When the SCL processed these samples, small-volume (1 L) splits of whole water and filtered water were prepared that were subsequently shipped to the NWQL for analysis of TKN, $\mathrm{NO}_{\mathrm{x}}, \mathrm{TN}-\mathrm{A}, \mathrm{DN}$, and PN. The SCL provided an alternative analysis of $\mathrm{PN}$ using dried and sieved sediment samples (50 to $75 \mathrm{mg}$, typically), which were separated from the large-volume water samples by continuousflow centrifugation. Two estimates of TN-C were calculated: one using PN from the NWQL and the other using PN from the SCL. In general, TN-K was slightly lower than TN-C, and TN-A also was lower than TN-C but by a greater amount (table 1). Reagent limitation from carbon or mineral particulates in the whole-water samples was suspected to be the cause of the negative bias in TN-A.

\section{Follow-Up Studies}

By the end of 2006, it had become apparent that some form of negative bias was present in TN-A data. However, the cause and extent of this bias, which was most apparent in samples with high SSCs, remained unknown. Therefore, a controlled-laboratory experiment, the results of which are described in this report, was undertaken to identify the cause and extent of the bias. Based on the results of these experiments, the Office of Water Quality issued a statement alerting USGS data users about potential negative bias in TN results from the alkaline-persulfate digestion method (U.S. Geological Survey, 2009). In response, a 2-year synopticfield study was conducted using data collected at 77 USGS stream-sampling sites to assess the magnitude of bias in environmental samples and to evaluate mitigation alternatives. This field study is described in this report and was considered "synoptic" in that it utilized data from a broad area.

\section{Purpose and Scope}

This report describes the performance of alkaline-persulfate and Kjeldahl digestion methods for assessment of TN by TN-A and TN-K approaches. These methods are compared to a third approach that computes $\mathrm{TN}$ as the sum of dissolved and particulate nitrogen analyses or TN-C. Methods and results are described for two complementary comparative studies that began with a series of laboratory experiments performed in 2007 and concluded with a comprehensive synoptic-field study conducted between June 2009 and September 2010. The synoptic-field study provided 905 samples from established NAWQA, NASQAN, and National Monitoring Network (NMN) (http://water.usgs.gov/nasqan/objectives_subnets.html) sites for which TN-A and TN-K values were evaluated in relation to TN-C. Bias and precision associated with each of the three approaches are characterized. The report concludes with a discussion of some advantages and disadvantages associated with three different scenarios for reducing potential bias in future data-collection efforts.

\section{Methods}

Bias and precision in estimates of total nitrogen were evaluated through laboratory experimentation as well as a synoptic-field study. Though bias was evaluated for TN-A and $\mathrm{TN}-\mathrm{K}$, the primary focus of both the laboratory experiment and the field study was to characterize the effect of sediment on TN-A bias.

Standards of known TN and sediment concentrations were used in laboratory experiments to verify the presence of bias in TN-A estimates of total nitrogen. In these experiments, increasing amounts of sediment reference materials were added to a deionized water matrix containing constant amounts of dissolved inorganic and organic nitrogen. Under these controlled conditions, it was possible to assess the extent of negative bias in TN-A in relation to the added mass of reference material and to distinguish among possible mechanisms for observed negative bias - alkaline-persulfate reagent limitation, reference-material constituents that were refractory (resistant to alkaline-persulfate digestion), or post-digestion interference in colorimetric determinative methods. Because TKN also was measured on the synthetic samples, it was possible to assess the magnitude of bias in TN-K over a range of sediment concentrations.

The subsequent synoptic-field study was designed to provide insights into the extent of bias expected for different TN-estimation approaches and how reduction in bias might affect the precision of reported TN. The designs and methods 


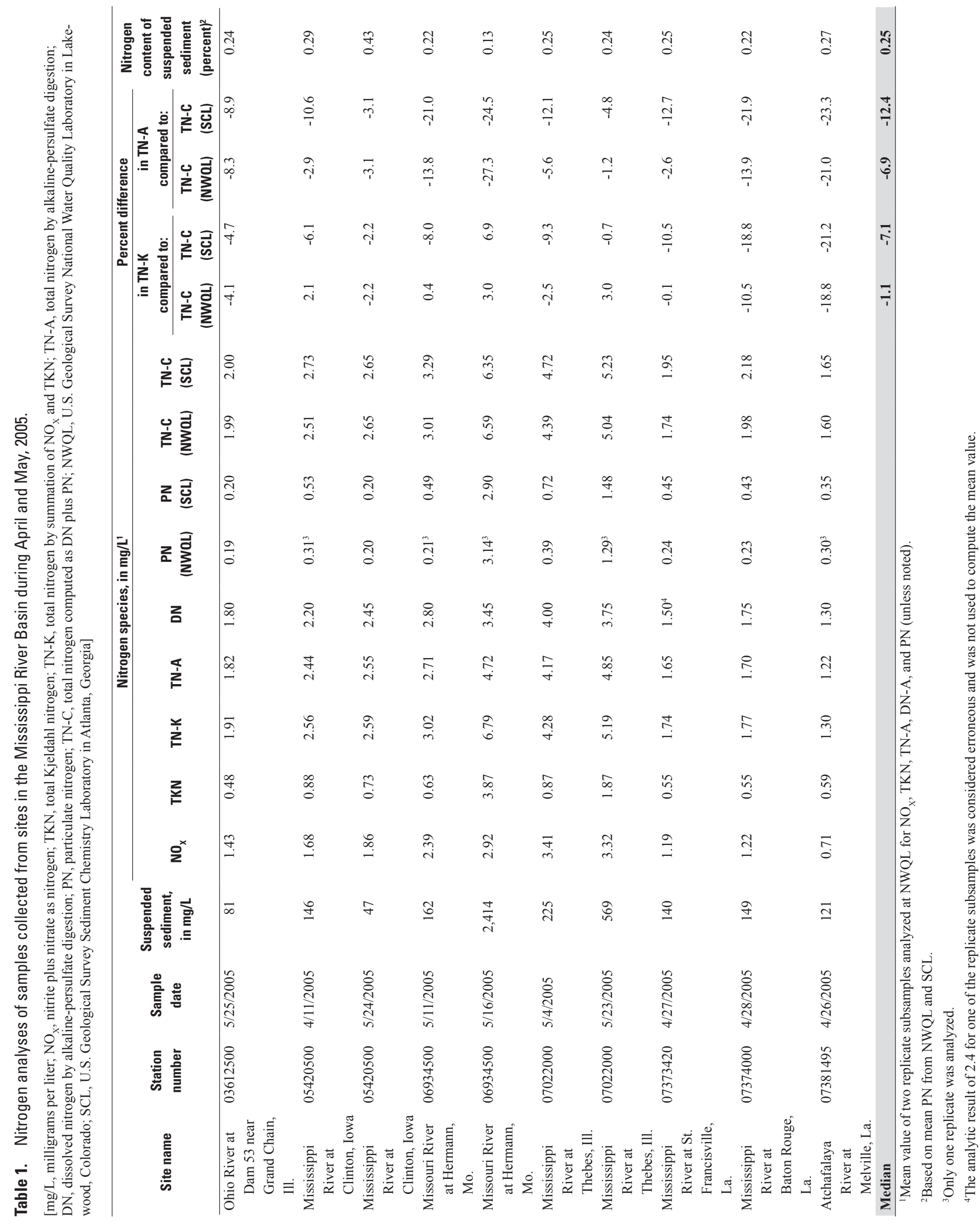


used for the laboratory experiment and the field study are described in subsections that follow. The three TN-estimation approaches also are summarized in the Glossary at the front of this report.

\section{Laboratory-Experiment Design and Sample Analysis}

In 2007, a controlled experiment explored potential causes of method bias in NWQL TN results. This laboratory experimentation addressed the following questions:

1. Is TN-A negatively biased in the presence of suspended sediment?

2. If so, does negative bias increase as SSC increases?

3. Is there a SSC threshold below which TN-A values are unbiased?

4. If the negative bias in TN-A values are caused by reagent limitation, could the bias be resolved by diluting wholewater samples prior to alkaline-persulfate digestion, increasing the concentration of persulfate in the digestion reagent, or both?

A secondary objective of the laboratory experiment was to determine whether TKN was positively biased, possibly because of conversion of nitrate to ammonia during the Kjeldahl digestion process. If this did happen, some nitrate would be counted twice when summing TKN and $\mathrm{NO}_{\mathrm{X}}$ to calculate TN-K.

The laboratory experiment was designed and coordinated by personnel in the USGS Branch of Quality Systems (BQS). All analyses were performed at the NWQL. The BQS prepared sediment-water samples by adding specific masses of standard reference materials to a fixed volume of nutrient-fortified, deionized water. A large batch of water matrix was prepared by adding nitrate and glycine to deionized water at nominal concentrations of 2.0 and $0.3 \mathrm{mg} / \mathrm{L}$ as $\mathrm{N}$, respectively. The former approximates the median nitrate concentration of the 10 samples analyzed in the 2005 field test (table 1). Glycine was used as a surrogate for the DON measured in samples from the 2005 field test. Sulfuric acid (having strength of 4.5 normality) was added to match field preservation of samples collected for total-nitrogen analysis. Ten milliliters $(\mathrm{mL})$ of this acidified, nitrogen-fortified, deionized-water matrix were pipetted into alkaline-persulfate or Kjeldahl-nitrogen digestion tubes, and then reference material was added in amounts corresponding to sediment concentrations ranging from $100 \mathrm{mg} / \mathrm{L}$ to $3,000 \mathrm{mg} / \mathrm{L}$. Replicate samples were prepared for each digestion method at each concentration.

Three reference materials were used for sediment amendments: Buffalo River sediment (standard reference material 2704) and Montana soil (standard reference material 2710), both of which are available from the National Institute of Standards and Technology (NIST), and Green River Shale (reference material SGR-1), which is a USGS geochemical reference material collected from the Mahogany zone of the Green River Formation. Buffalo River sediment and Montana soil were selected as reasonable surrogates for suspended sediment in riverine systems; the mass percentage of nitrogen content of these materials is in the range observed for suspended sediment at NASQAN sites in the 2005 field test (table 1). Green River Shale, which contains greater concentrations of nitrogen, was selected to test for reagent limitation in alkaline-persulfate and Kjeldahl digestion procedures. These three materials lack NIST-certified values for nitrogen, so reference values were determined by multiple analyses of each material at the SCL (table 2). Each analysis was done on about 200-250 mg of material. Analyses of Montana soil and Green River Shale were done routinely over the time period 2000-07 and analyses of Buffalo River sediment were done in 2007 in preparation for the laboratory experiment. Mean nitrogen content was established as 0.20 percent by weight $(0.20 \mathrm{mg} \mathrm{N}$ per $100 \mathrm{mg}$ sediment) in Buffalo River sediment, 0.13 percent in Montana soil, and 0.90 percent in Green River Shale. The value determined for Buffalo River sediment is close to the nitrogen content ( 0.191 percent) recently reported by the Maryland Geological Survey (Wells and Ortt, 2011, p. 30), and the value determined for Montana soil is identical to the nitrogen content reported by the Keck Laboratory at Arizona State University (http://kfleb.asu.edu/Analytical/gIRMS/ Instrumentandanalysis/Analytical/CandNonEA.html).

Prior to the laboratory experiment, the three reference materials were analyzed at the NWQL (table 3 ) to compare nitrogen contents to the reference values determined by the SCL and to evaluate whether results were consistent across a range of material mass analyzed. As shown in figure 1, the

Table 2. Nitrogen content of reference materials determined by analyses at the U.S. Geological Survey Sediment Chemistry Laboratory, Atlanta, Georgia.

[NIST, National Institue of Standards and Technology; SRM, Standard Reference Material; USGS, U.S. Geological Survey; SGR-1, USGS Green River Shale]

\begin{tabular}{llcccc}
\hline \multicolumn{1}{c}{ Reference material } & Reference identifier & $\begin{array}{c}\text { Number of } \\
\text { analyses }\end{array}$ & $\begin{array}{c}\text { Mean nitrogen content } \\
\text { (percent) }\end{array}$ & $\begin{array}{c}\text { Standard deviation } \\
\text { (percent) }\end{array}$ & $\begin{array}{c}\text { Relative standard deviation } \\
\text { (percent of mean) }\end{array}$ \\
\hline Buffalo River sediment & NIST SRM 2704 & 10 & 0.203 & 0.004 & 2.2 \\
Montana soil & NIST SRM 2710 & 61 & 0.130 & 0.023 & 17.5 \\
Green River Shale & USGS SGR-1 & 50 & 0.901 & 0.039 & 4.4 \\
\hline
\end{tabular}




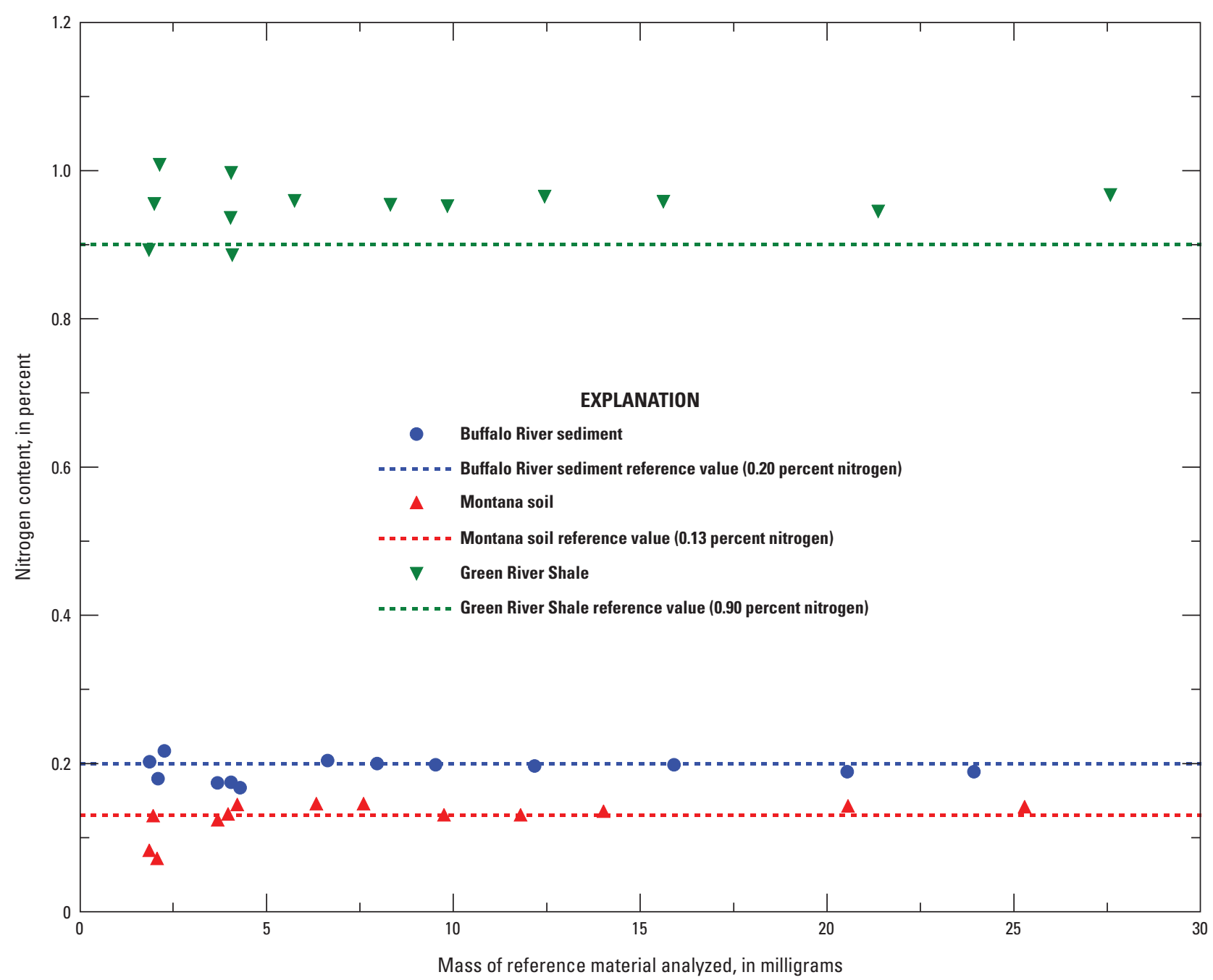

Figure 1. Nitrogen content measured for various amounts of three reference materials at the U.S. Geological Survey National Water Quality Laboratory, Denver, Colorado, in comparison to reference values determined at the U.S. Geological Survey Sediment Chemistry Laboratory, Atlanta, Georgia.

NWQL results for Buffalo River sediment and Montana soil agree closely with the reference values over a range of mass that covers the planned reference-material additions to laboratory samples. For these two reference materials, nitrogencontent results from the NWQL generally were unbiased with only a small variability over most of the range. Results for Green River Shale had a positive bias of about 6 percent and a slightly higher variability than for the other two materials; however, this was not a major concern for the laboratory experiment because this shale material had been selected to represent extreme conditions not expected in riverine sediments.

The expected or "true" concentration of nitrogen in each synthetic sample of fortified water plus sediment was the sum of the known amount of dissolved nitrate and organic nitrogen (glycine) in the aqueous matrix plus PN known from the mass of reference material added to each digestion tube. PN concentration was determined as: where

$$
P N=\frac{\left(\begin{array}{l}
N_{p c t} / 100 \\
V o l
\end{array}\right) \operatorname{Sed}}{1,000}
$$

$N_{p c t} \quad$ is the mass percentage of nitrogen in the reference material,

Sed is the mass, in milligrams, of reference material added to each digestion tube, and

$\mathrm{Vol}$ is the volume, in milliliters, of nutrientfortified matrix into which the reference material was added.

TN-A results were compared to the known concentrations of total nitrogen. TKN results were compared to the known concentrations of organic nitrogen plus PN.

To test the effect of sample dilution and reagent enhancement using the alkaline-persulfate method, some digests for $\mathrm{TN}-\mathrm{A}$ analysis were prepared with half the aqueous-matrix volume and double the persulfate reagent volume. This 
Table 3. Nitrogen content of reference materials determined by analyses at the U.S. Geological Survey National Water Quality Laboratory in Denver, Colorado.

[mg, milligrams; --, not applicable]

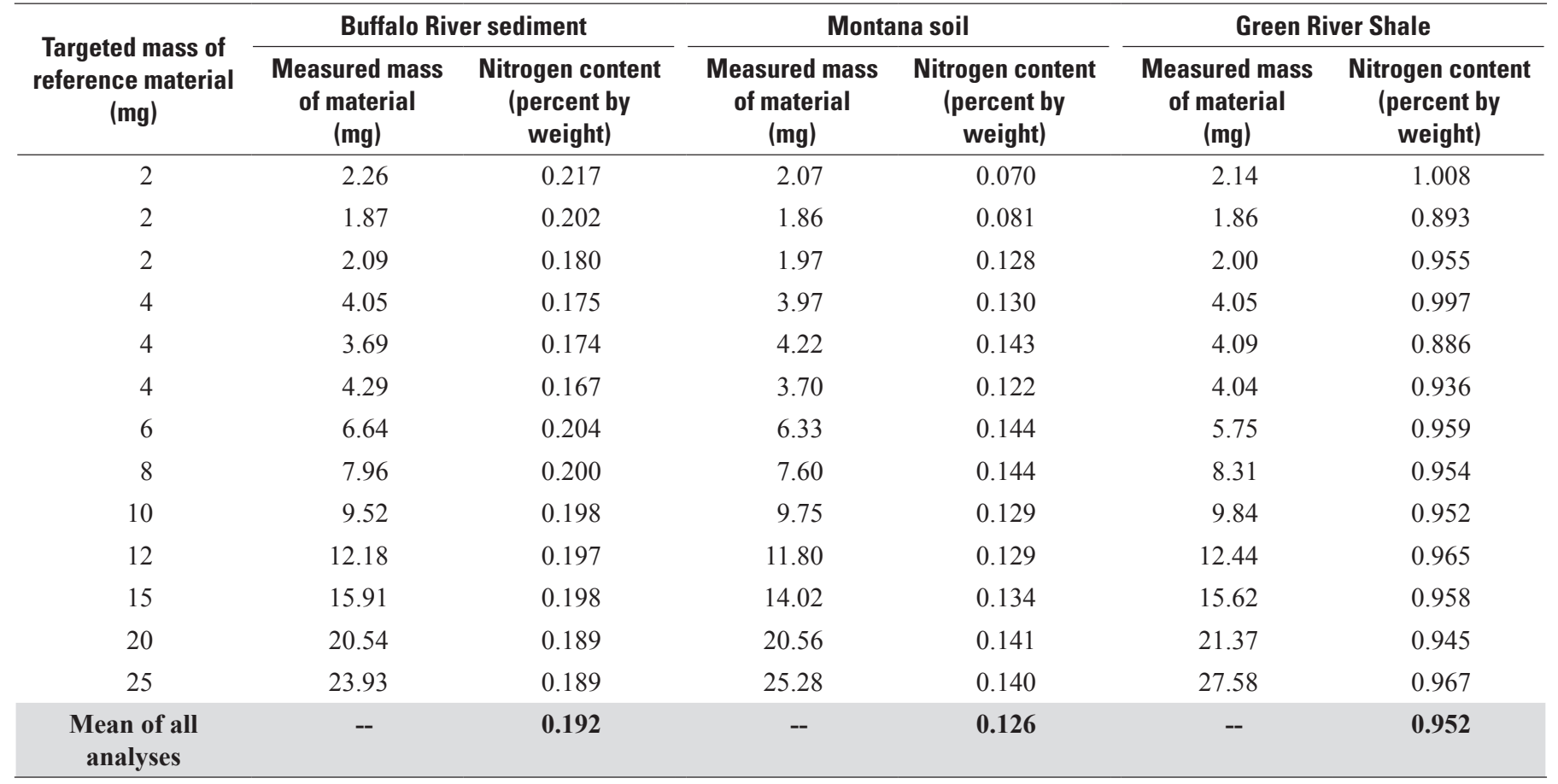

increased the reagent concentration by a factor of four relative to sample volume, which theoretically would decrease the effect of reagent limitation.

For each synthetic sample, the expected concentrations of selected nitrogen species and measured concentrations of TN-A and TKN are listed in the appendix (tables A1 and A2, respectively). Bias, relative bias (in percent), and apparent recoveries are also listed in the appendix.

\section{Synoptic-Field-Study Design and Sample Analysis}

The synoptic-field study was designed not only to assess the extent of bias in TN-A and TN-K but also to evaluate the efficacy of using the sum of DN and PN (TN-C) as a more accurate alternative to direct measurement of total nitrogen in water samples. Data were collected to assess how variability in analytical results for PN might affect bias and precision of TN-C values. To this end, replicate filter pads for PN determinations were prepared for each sample to permit assessment of PN variability over the full range of SSC. Besides data collected during the synoptic-field study, additional data are stored in the NWIS database that could be used to make TN-A and TN-K comparisons; however, because sampling protocols and analytical methods associated with these data were not explicitly defined, they are not evaluated in this report.
From June 2009 through September 2010, analytical schedules of three national monitoring programs-NASQAN, NAWQA, and NMN - were modified to include alkalinepersulfate digestion for determining DN and HTCO for PN, which allowed for computation of TN-C values. Samples included in the synoptic-field study were collected at 77 sites (fig. 2, table 4) using standard procedures (U.S. Geological Survey, variously dated). TN-A data were being collected already or were added to the samples at all 77 sites. TN-K data also were available at 19 of these 77 sites and were typically associated with sites of the NASQAN and NMN programs. Subsamples for particular analyses or suites of analyses were prepared using churn splitters or cone splitters (U.S. Geological Survey, variously dated, section 5.1.1). The purpose of the synoptic-field study was twofold: (1) to evaluate the extent of TN-A and TN-K bias in environmental samples; and (2) to evaluate potential alternatives to the existing methods for estimating TN concentrations.

\section{Dissolved-Nitrogen Procedures}

DN subsamples were processed at collection sites by filtering water through a polysulfone, pleated membrane having $0.45-\mu \mathrm{m}$ pore size. Filtrates were bottled, chilled, and sent to the NWQL for analysis by USGS alkaline-persulfate digestion method I-2650-03 (Patton and Kryskalla, 2003). 


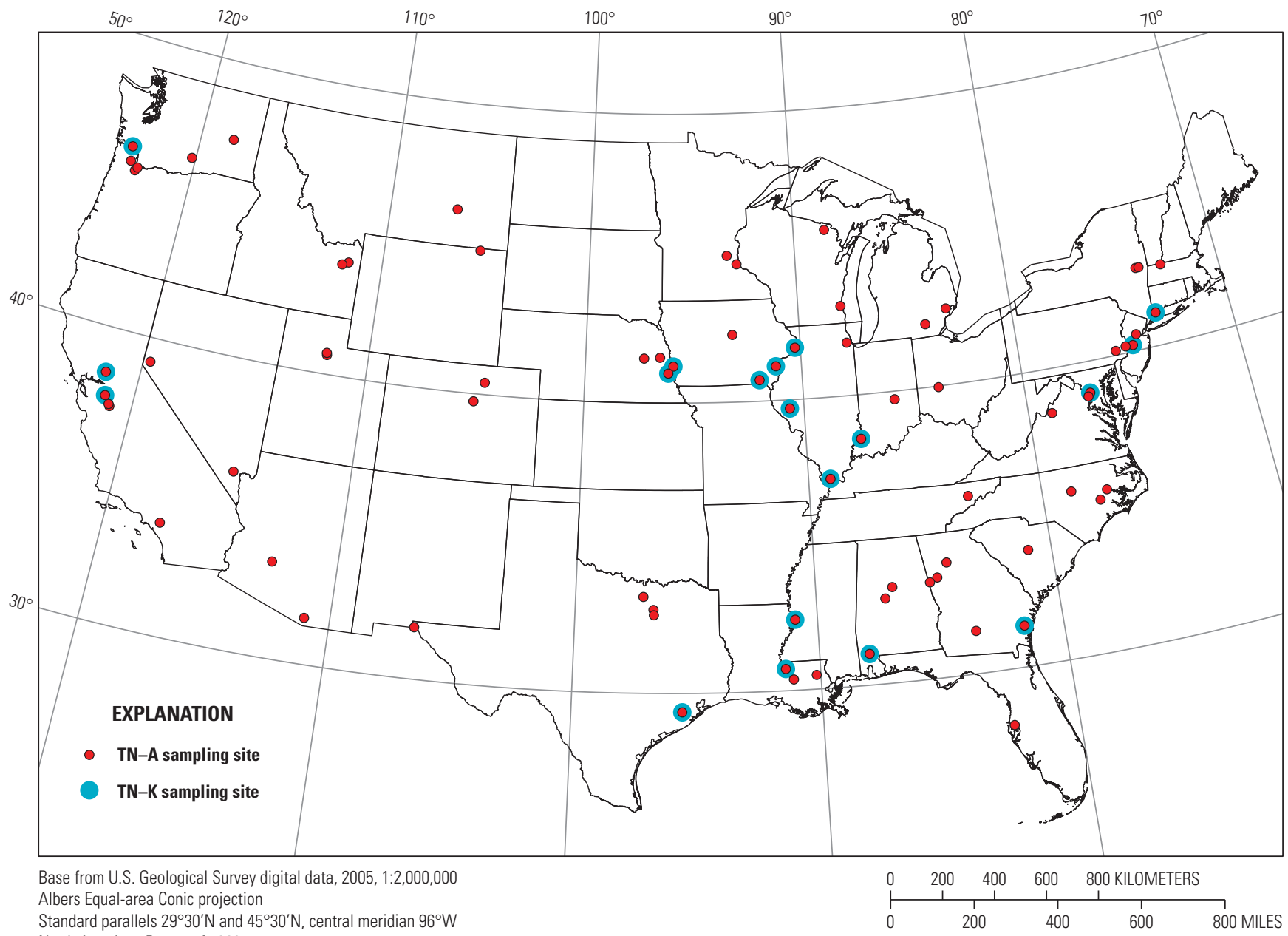

North American Datum of 1983

Figure 2. Distribution of sites sampled for the synoptic-field study of bias in total nitrogen by alkaline-persulfate digestion (TNA) and as the sum of total Kjeldahl nitrogen and nitrate plus nitrite (TN-K), 2009-10.

\section{Particulate-Nitrogen Procedures}

PN subsamples were collected on 25-mm diameter, $0.7-\mu \mathrm{m}$ pore size glass-fiber filter pads by vacuum filtration using procedures specified for total particulate carbon in the USGS National Field Manual (U.S. Geological Survey, variously dated, section 5.2.2.C). The filtration apparatus consisted of a $25-\mathrm{mm}$ polysulfone filter funnel with a $200-\mathrm{mL}$ reservoir and stainless steel filter support. It was connected to a 500$\mathrm{mL}$ polypropylene filtering flask and a metered, hand-vacuum pump was used to induce a vacuum of -68.9 kilopascals $(-10$ pound-force per square inch) or less. A subsample was split from the sample into a baked, glass bottle. The subsample was shaken vigorously to suspend the particulate matter before pouring an aliquot into the filter funnel, equipped with a filter pad, and applying vacuum to the filtering funnel. The filtrate was collected and measured in a polypropylene graduated cylinder. In some cases, the filtrate volume was measured using the graduated cylinder (or a graduated, polystyrene pipette for heavily sediment-laden samples) prior to being added to the filter funnel. After sample volume was recorded, inorganic-free water was used to rinse any residual sediments adhering to the walls of the cylinder, pipette, or filter funnel onto the filter. After filtration was complete, as indicated by a visibly dry filter, the system was depressurized and the top of the filter funnel was carefully removed to expose the filter. Using two metal forceps, the filter was gently removed from the support, placed on a clean aluminum foil square, and folded in half with the suspended material on the inside. The filters were enclosed within aluminum foil envelopes, chilled, and sent to the NWQL for analysis.

Because clogging increases as more whole water is filtered, PN sample volumes necessarily decrease as particulate concentrations increase. Although 50-mL PN aliquots were typical, 2-mL aliquots were used for some sediment-laden samples. Precision of PN values resulting from 2-mL aliquots was expected to be inferior to that derived from $50-\mathrm{mL}$ (or greater) aliquots. Therefore, extra efforts were made to quantify PN variability. Specifically, up to four replicate PN filters were prepared for each sample. In most cases, the filtered volumes were the same for each replicate. Regardless, filtered volumes for each replicate filter were recorded. 
Table 4. Sampling sites and number of samples collected for the synoptic-field study of bias in total nitrogen as determined by alkaline-persulfate digestion (TN-A) or the sum of total Kjeldahl nitrogen and nitrate plus nitrite (TN-K), 2009-10.

[Station ID, U.S. Geological Survey station identification number; TN-A, total nitrogen measured from alkaline-persulfate digestion; TN-K, total nitrogen computed as the sum of total Kjeldahl nitrogen and nitrate plus nitrite; --, not available]

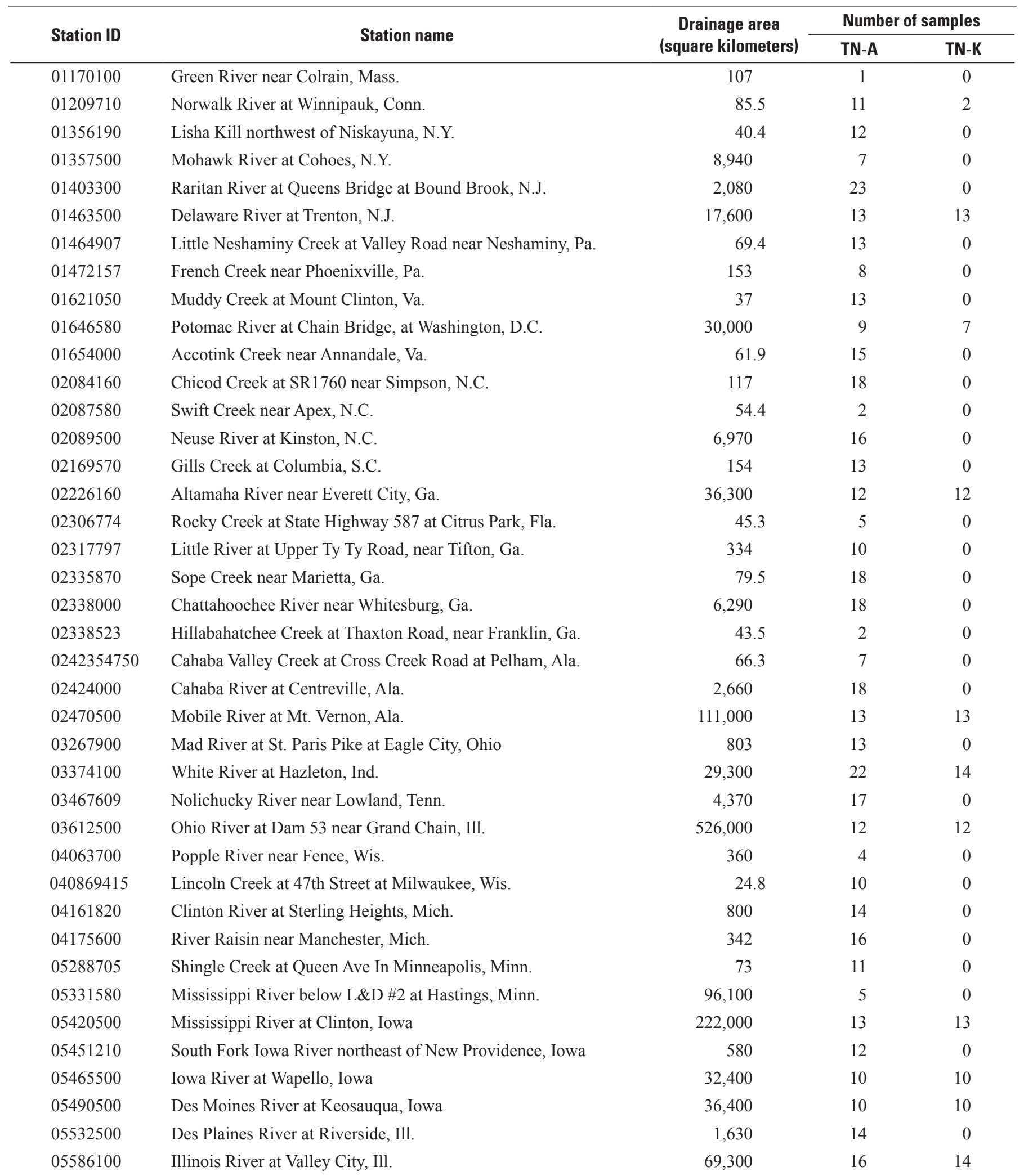


Table 4. Sampling sites and number of samples collected for the synoptic-field study of bias in total nitrogen as determined by alkaline-persulfate digestion (TN-A) or the sum of total Kjeldahl nitrogen and nitrate plus nitrite (TN-K), 2009-10. - Continued

[Station ID, U.S. Geological Survey station identification number; TN-A, total nitrogen measured from alkaline-persulfate digestion; TN-K, total nitrogen computed as the sum of total Kjeldahl nitrogen and nitrate plus nitrite; --, not available]

\begin{tabular}{|c|c|c|c|c|}
\hline \multirow{2}{*}{ Station ID } & \multirow{2}{*}{ Station name } & \multirow{2}{*}{$\begin{array}{l}\text { Drainage area } \\
\text { (square kilometers) }\end{array}$} & \multicolumn{2}{|c|}{ Number of samples } \\
\hline & & & TN-A & TN-K \\
\hline 06295000 & Yellowstone River at Forsyth, Mont. & 104,000 & 2 & 0 \\
\hline 06324970 & Little Powder River above Dry Creek, near Weston, Wyo. & 3,200 & 4 & 0 \\
\hline 06610000 & Missouri River at Omaha, Nebr. & 836,000 & 10 & 10 \\
\hline 06713500 & Cherry Creek at Denver, Colo. & 1,060 & 11 & 0 \\
\hline 06754000 & South Platte River near Kersey, Colo. & 25,000 & 14 & 0 \\
\hline 06795500 & Shell Creek near Columbus, Nebr. & 761 & 13 & 0 \\
\hline 06800000 & Maple Creek near Nickerson, Nebr. & 953 & 16 & 0 \\
\hline 06805500 & Platte River at Louisville, Nebr. & 221,000 & 18 & 14 \\
\hline 07288955 & Yazoo River below Steele Bayou near Long Lake, Miss. & 34,600 & 23 & 11 \\
\hline 07373420 & Mississippi River near St. Francisville, La. & $2,910,000$ & 14 & 14 \\
\hline 07375050 & Tchefuncte River near Covington, La. & 376 & 14 & 0 \\
\hline 07379960 & Dawson Creek at Bluebonnet Blvd near Baton Rouge, La. & 39.1 & 10 & 0 \\
\hline 08051500 & Clear Creek near Sanger, Tex. & 764 & 13 & 0 \\
\hline 08057200 & White Rock Creek at Greenville Ave, Dallas, Tex. & 172 & 6 & 0 \\
\hline 08057410 & Trinity River below Dallas, Tex. & 16,300 & 17 & 0 \\
\hline 08116650 & Brazos River near Rosharon, Tex. & 117,000 & 13 & 13 \\
\hline 08364000 & Rio Grande at El Paso, Tex. & 83,400 & 12 & 0 \\
\hline 094196783 & $\begin{array}{l}\text { Las Vegas Wash below Flamingo Wash confluence near } \\
\text { Las Vegas, Nev. }\end{array}$ & 3,550 & 3 & 0 \\
\hline 09481740 & Santa Cruz River at Tubac, Ariz. & 3,130 & 11 & 0 \\
\hline 09517000 & Hassayampa River near Arlington, Ariz. & 3,810 & 7 & 0 \\
\hline 10168000 & Little Cottonwood Creek at Jordan River near Salt Lake City, Utah & 119 & 5 & 0 \\
\hline 10171000 & Jordan River at 1700 South at Salt Lake City, Utah & 8,900 & 11 & 0 \\
\hline 10311400 & Carson River at Deer Run Road near Carson City, Nev. & 2,480 & 14 & 0 \\
\hline 11074000 & Santa Ana River below Prado Dam, Calif. & 5,850 & 19 & 0 \\
\hline 11273500 & Merced River at River Road Bridge near Newman, Calif. & 3,300 & 7 & 0 \\
\hline 11274538 & Orestimba Creek at River Road near Crows Landing, Calif. & -- & 11 & 0 \\
\hline 11303500 & San Joaquin River near Vernalis, Calif. & 35,100 & 23 & 12 \\
\hline 11447650 & Sacramento River at Freeport, Calif. & -- & 13 & 13 \\
\hline 12464770 & Crab Creek at Rocky Ford Road near Ritzville, Wash. & 995 & 10 & 0 \\
\hline 12505450 & Granger Drain at Granger, Wash. & -- & 13 & 0 \\
\hline 13055000 & Teton River near St. Anthony, Idaho & 2,270 & 5 & 0 \\
\hline 13056500 & Henrys Fork near Rexburg, Idaho & 7,560 & 11 & 0 \\
\hline 14205400 & East Fork Dairy Creek near Meacham Corner, Oreg. & 87.5 & 4 & 0 \\
\hline 14206950 & Fanno Creek at Durham, Oreg. & 81.6 & 6 & 0 \\
\hline 14211720 & Willamette River at Portland, Oreg. & 29,000 & 19 & 0 \\
\hline 14246900 & Columbia River at Beaver Army Terminal near Quincy, Oreg. & 665,000 & 12 & 11 \\
\hline 394340085524601 & Sugar Creek at County Road 400S at New Palestine, Ind. & 240 & 15 & 0 \\
\hline
\end{tabular}


The NWQL analyzed each filter for particulate nitrogen using HTCO methods. The PN result from the first filter in each set was used to compute TN-C. For cases involving censored PN values, half of the method-detection level—or onefourth of the laboratory reporting level (Childress and others, 1999) — was used in the computation of TN-C. The additional replicate filters allowed characterization of the variability that might be introduced by the use of TN-C as an alternative method to TN-A or TN-K.

\section{Selection of a Total-Nitrogen Reference for Evaluation of Bias in Synoptic-Field Samples}

The rationale for using TN-C, the sum of DN and PN, as the least biased estimator of total nitrogen in synoptic study samples is threefold. First, in the absence of suspended sediment, DN method bias - from reagent limitation caused by dissolved inorganic and organic nitrogen, dissolved organic carbon, or both - is expected to be minor. Previous research at the NWQL reported recoveries for $2.5 \mathrm{mg} / \mathrm{L}$ as $\mathrm{N}$ of inorganic and organic nitrogen species - ammonia, urea, nicotinic acid, and glycine - in the range of 97.9 to 100.5 percent (Patton and Kryskalla, 2003, p. 21-22, table 12). These DN recoveries agree favorably with those reported for similar methods developed elsewhere (Hosomi and Sudo, 1986; Cabrera and Beare, 1993). Second, the onset of reagent limitation from dissolvedorganic carbon in the USGS DN method occurred at $150 \mathrm{mg} / \mathrm{L}$ as carbon, much greater than typically found in environmental water (Patton and Kryskalla, 2003, p. 21-22, fig. 5). And third, PN results from HTCO analyses at the NWQL had low variability and were nearly unbiased in comparison to reference values (fig. 1). Researchers concerned with total nitrogen determinations in estuarine and marine waters adopted this approach over 20 years ago on the basis of similar experiments and reasoning (Zimmerman and others, 1997).

\section{Bias and Precision Metrics}

Bias in the total-nitrogen methods was evaluated by comparing measured values with a reference value. As described earlier, the laboratory experiment used samples having known total-nitrogen concentration. Because the true TN concentration was unknown in the synoptic-field study samples, results from the TN-C method were used as reference values. Bias was computed as:

$$
\text { Bias }=T N_{\text {meas }}-T N_{\text {ref }}
$$

where
Bias
is the deviation of the measured value from the reference value, in milligrams per liter as $\mathrm{N}$;
$T N_{\text {meas }} \quad$ is the measured value of TN (either TN-A or $\mathrm{TN}-\mathrm{K}$ ), in milligrams per liter as $\mathrm{N}$; and
$T N_{\text {ref }} \quad$ is the reference value of TN (known TN for the laboratory experiment or TN-C for the synoptic-field study), in milligrams per liter as $\mathrm{N}$.

Bias is positive if measured TN exceeds the expected concentration (reference value); bias is negative if measured TN is less than the expected concentration. Because bias could vary over several orders of magnitude, "relative bias" was computed to normalize Bias relative to the reference value:

$$
\text { Relative Bias }=100 \times \text { Bias } / T N_{\text {ref }}
$$

where

Relative Bias is the difference between the measured and reference $\mathrm{TN}$, in percent.

The precision of an analytical result is affected by variability introduced during the collection of field samples as well as by variability that is inherent in all analytical methods. Variability is determined by repeated, independent measurements of analyte concentrations in replicate samples. Measures of variability include the standard deviation and relative standard deviation (RSD) of these measurements. Standard deviation of nutrient analytical results, based on field replicates, generally is uniform near the analytical detection limit, but at concentrations higher than about 5-10 times the detection limit, standard deviation tends to increase in proportion to concentration (Mueller and Titus, 2005). Within this higher range of concentrations, RSD (defined as the standard deviation of the set of replicates divided by the mean concentration) generally is uniform.

\section{Assessment of Precision for Total- Nitrogen Methods}

For each measured-nitrogen constituent, precision was characterized by determining variability over low and high concentration ranges. Variability was compared among constituents, and the effect of variability on calculated values of $\mathrm{TN}$ was estimated.

\section{Precision of Nitrogen Analyses}

During the synoptic-field study, 67 replicate water samples were collected and analyzed for at least one of the constituents $\mathrm{NO}_{\mathrm{X}}, \mathrm{DN}$, or TN-A. One or more replicate filter samples for PN analysis were collected at 896 of the 905 sampling events.

For this report, unrounded and uncensored data were obtained from the NWQL for analysis of replicate variability. This was done to avoid problems that could be introduced when comparing small differences between two rounded values or when comparing censored values to each other or to uncensored values. These data are listed in the appendix 
Table 5. Estimates of variability in replicate measurements for nitrogen species.

[mg/L, milligrams per liter; $\mathrm{N}$, nitrogen]

\begin{tabular}{|c|c|c|c|c|c|c|c|}
\hline \multirow[b]{2}{*}{ Analyte } & \multirow[b]{2}{*}{ Source } & \multicolumn{3}{|c|}{ Low concentrations } & \multicolumn{3}{|c|}{ High concentrations } \\
\hline & & $\begin{array}{l}\text { Concentra- } \\
\text { tion range } \\
(\mathrm{mg} / \mathrm{L})\end{array}$ & $\begin{array}{l}\text { Number of } \\
\text { replicate } \\
\text { sets }\end{array}$ & $\begin{array}{l}\text { Variability } \\
\text { (mean standard } \\
\text { deviation, } \\
\text { in } \mathrm{mg} / \mathrm{L} \text { ) }\end{array}$ & $\begin{array}{l}\text { Concentra- } \\
\text { tion range } \\
\text { (mg/L) }\end{array}$ & $\begin{array}{c}\text { Number of } \\
\text { replicate } \\
\text { sets }\end{array}$ & $\begin{array}{c}\text { Variability } \\
\text { (mean relative } \\
\text { standard deviation } \\
\text { in percent) }\end{array}$ \\
\hline \multirow[t]{2}{*}{$\begin{array}{l}\text { Nitrite plus nitrate, } \\
\text { as N }\end{array}$} & $\begin{array}{l}\text { Synoptic-field study, } \\
2009-10\end{array}$ & $0.01-0.3$ & 14 & 0.001 & $0.3-15$ & 49 & 1.0 \\
\hline & $\begin{array}{l}\text { Mueller and Titus, } \\
2005\end{array}$ & $0.004-1.0$ & 691 & 0.012 & $1.0-41$ & 541 & 2.2 \\
\hline Dissolved nitrogen & $\begin{array}{l}\text { Synoptic-field study, } \\
2009-10\end{array}$ & -- & -- & -- & $0.2-15$ & 51 & 2.3 \\
\hline Total nitrogen & $\begin{array}{l}\text { Synoptic-field study, } \\
2009-10\end{array}$ & -- & -- & -- & $0.2-15$ & 62 & 2.1 \\
\hline Particulate nitrogen & $\begin{array}{l}\text { Synoptic-field study, } \\
2009-10\end{array}$ & $0.005-0.05$ & 171 & 0.007 & $0.051-20$ & 725 & 13 \\
\hline Total Kjeldahl nitrogen & $\begin{array}{l}\text { Mueller and Titus, } \\
2005\end{array}$ & $0.05-0.5$ & 523 & 0.027 & $0.5-40$ & 591 & 7.6 \\
\hline
\end{tabular}

(table A3). Because data have not been quality assured using laboratory rounding and censoring rules, readers are cautioned these data should not be used for purposes other than precision analyses such as done for this report. Uncensored results could include negative values because of uncertainty in the calibration curve at very low concentrations. This explains the two replicate data values for PN that have concentrations of -0.002 and $-0.020 \mathrm{mg} / \mathrm{L}$ as $\mathrm{N}$ in table $\mathrm{A} 3$.

Data plotted in figure 3 illustrate the low-range and high-range variability for $\mathrm{NO}_{\mathrm{x}}, \mathrm{DN}, \mathrm{TN}$, and PN. These plots include a locally weighted scatterplot-smoothing (LOWESS) curve (Helsel and Hirsch, 2002) through the data points to show the general relation between concentration and standard deviation or RSD. Where this curve is horizontal, there is no relation between standard deviation or RSD and concentration, so variability could be considered constant. The boundary concentration was determined visually by selecting a point on the mean-concentration axis below which the curve for standard deviation was essentially horizontal and above which the curve for RSD was essentially horizontal. The division between low- and high-concentration ranges is represented with a vertical dashed line. Variability could then be estimated as the mean standard deviation for the low range or the mean RSD for the high range.

Variability for $\mathrm{NO}_{\mathrm{x}}$ (figs. $3 A$ and $3 B$ ) has a division at a concentration of $0.3 \mathrm{mg} / \mathrm{L}$ as $\mathrm{N}$ (table 5 ). Over the low range, variability is estimated as the mean standard deviation (plus or minus $( \pm) 0.001 \mathrm{mg} / \mathrm{L}$ as $\mathrm{N}$ ), and over the high range, as the mean RSD ( \pm 1.0 percent of the measured concentration). Both these estimates are lower than those reported by Mueller and Titus (2005) using a dataset with many more samples and a larger overall range of $\mathrm{NO}_{\mathrm{X}}$ concentrations. The true variability of $\mathrm{NO}_{\mathrm{X}}$ might be slightly underestimated using replicates from the 2009-10 field study, but there is no indication that variability is larger than the previous estimate from Mueller and Titus.

Plots of standard deviation and RSD for DN and TN show no obvious division between low- and high-range variability (figs. $3 C-F$ ). The trend in RSD is about constant for both of these analytes; therefore, variability could be estimated by the average RSD for all concentrations greater than $0.2 \mathrm{mg} / \mathrm{L}$ as $\mathrm{N}$. In general, estimated variability is low (typically no more than 2 percent) for measured values of DN, TN, and $\mathrm{NO}_{\mathrm{X}}$ in excess of $1 \mathrm{mg} / \mathrm{L}$ as $\mathrm{N}$.

Many more replicates were collected for PN than for the other analytes during the synoptic-field study. This was done because there was little previous information about the variability of the PN method. The plots showing the relations of standard deviation and RSD with mean PN concentration were slightly truncated in order to more clearly show patterns in variability (figs. $3 G$ and $3 H$ ). Four high standard-deviation values $(0.5-2.0 \mathrm{mg} / \mathrm{L}$ as $\mathrm{N}$ ) and one high RSD value (about 300 percent) are not shown, as are three sets of replicates with mean concentrations less than $0.01 \mathrm{mg} / \mathrm{L}$ as $\mathrm{N}$ (the lowest detection limit for PN during the study period). All values are retained in determining the LOWESS lines shown on the plots. There is no obvious threshold dividing low and high ranges of variability. Standard deviation is fairly constant up to a concentration of $0.05 \mathrm{mg} / \mathrm{L}$ as $\mathrm{N}$ but does not increase substantially until concentrations become greater than about $0.3 \mathrm{mg} / \mathrm{L}$ as $\mathrm{N}$. There is an inflection point in the RSD smoothing line at about $0.05 \mathrm{mg} / \mathrm{L}$ as $\mathrm{N}$, but it is not horizontal over any of the range. For mean PN concentrations no greater than $0.05 \mathrm{mg} / \mathrm{L}$ as $\mathrm{N}$ (approximately 5 times the lowest detection limits), variability averaged $\pm 0.007 \mathrm{mg} / \mathrm{L}$ as N, which is between the two variability estimates for the low range of $\mathrm{NO}_{\mathrm{X}}$ concentrations (table 5). Over the high range (mean PN greater than $0.05 \mathrm{mg} / \mathrm{L}$ as $\mathrm{N}$ ), mean RSD is \pm 13 percent of 

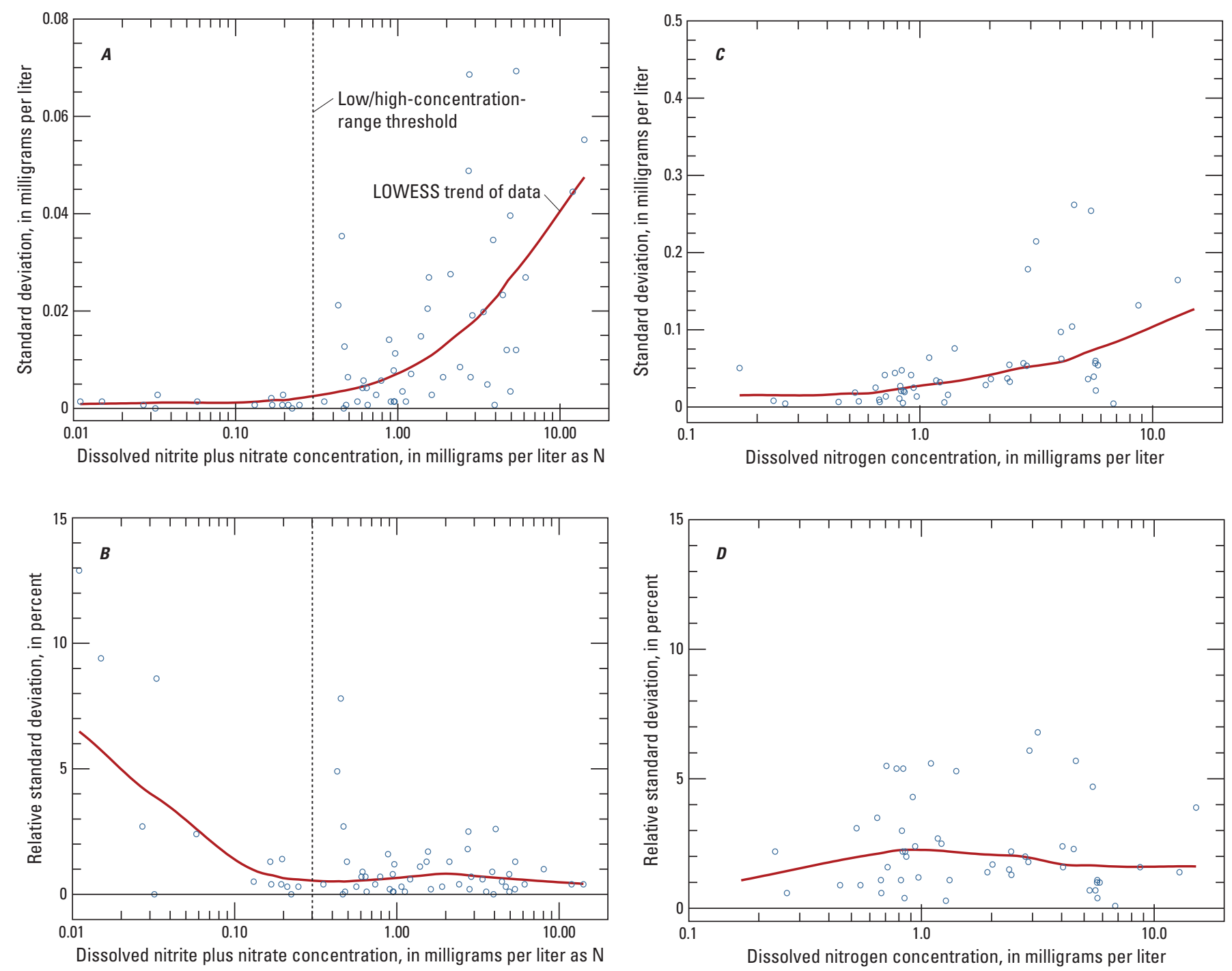

Figure 3. Relations of standard deviation $(A, C, E$, and $G)$ and relative standard deviation $(B, D, F$, and $H)$ to mean concentration for selected nitrogen species in replicate samples collected for the synoptic-field study, 2009-10. (LOWESS trend line is locally weighted scatterplot smooth.)

the measured concentration, larger than the high-range variability for other nitrogen species (table 5). RSD of individual replicate sets was much larger in many cases, though RSD was less than 20 percent for almost 80 percent of these sets, and RSD exceeded 30 percent in fewer than 8 percent of sets. For consistency with other constituents, variability for highconcentration PN was estimated as mean RSD, but this might be somewhat low under certain conditions.

Because TKN sampling occurred at only 16 of the 77 sites in the synoptic-field study, only 12 replicate samples were collected, and variability of TKN could not be estimated using those data. The estimates provided by Mueller and Titus (2005) are reproduced in table 5. Average RSD over the high range of concentrations is larger than the corresponding estimates for $\mathrm{NO}_{\mathrm{X}}, \mathrm{DN}$, and $\mathrm{TN}$ and is smaller than the estimate for PN.

\section{Factors That Could Affect the Variability of Particulate-Nitrogen Measurements}

Particulate nitrogen is derived from, and thus strongly correlated to, concentrations of suspended sediment. Thus, the variability of PN might be related to the variability of suspended sediment, especially if sample filtration is affected by high or low SSCs. This sampling issue is associated with the amount of water that is passed through the filter during collection of the particulates. At high SSCs, it is not possible to filter much water before clogging occurs. In this study, although most PN samples were collected from at least $50 \mathrm{~mL}$ of water, some were collected from as little as $2 \mathrm{~mL}$. The relation of SSC and filtered volume to the RSD of PN measurements in the synoptic-field-study dataset is plotted in figure 4. RSD is slightly higher at low SSCs and slightly lower at high SSCs, 

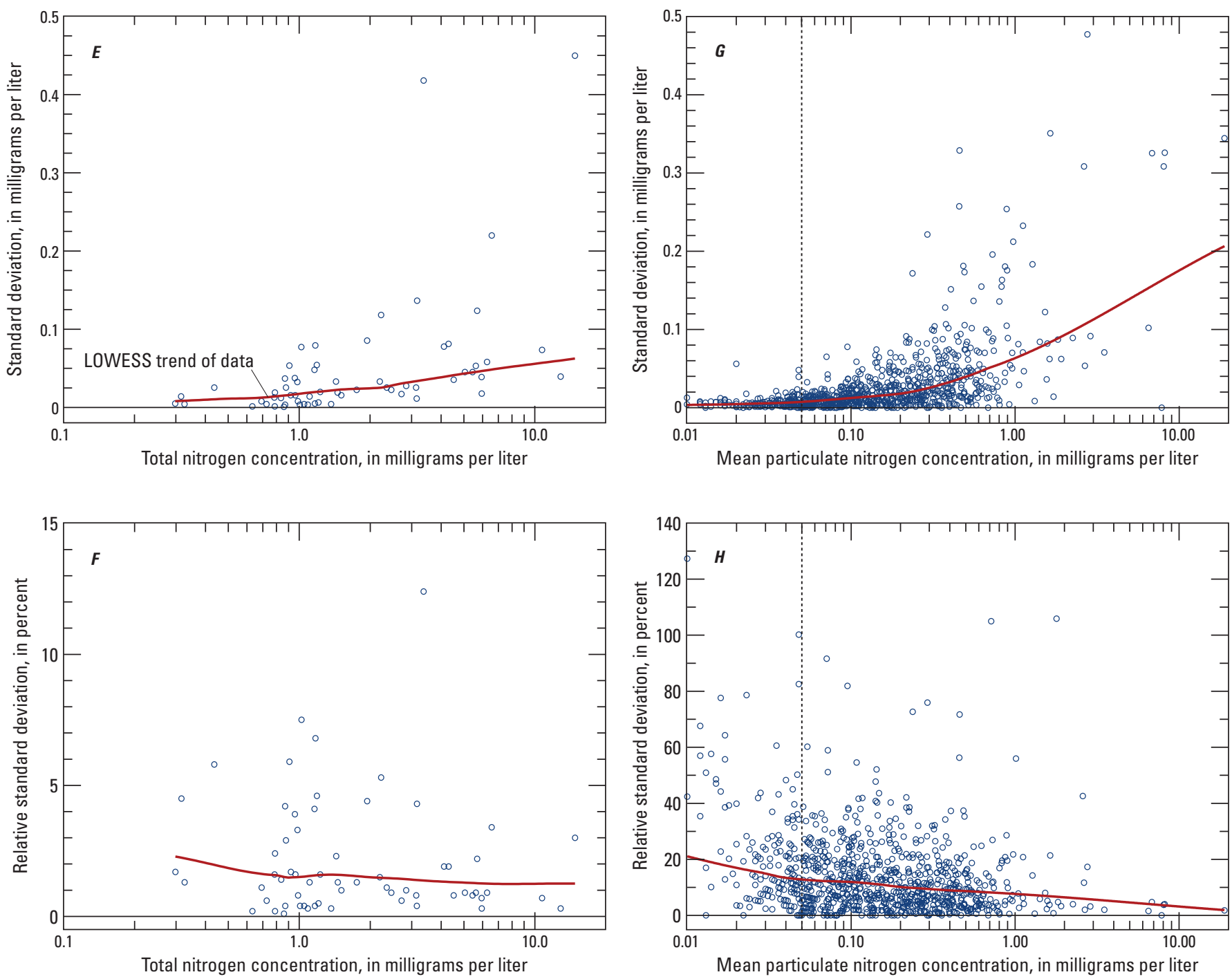

Figure 3. Relations of standard deviation $(A, C, E$, and $G)$ and relative standard deviation $(B, D, F$, and $H)$ to mean concentration for selected nitrogen species in replicate samples collected for the synoptic-field study, 2009-10. (LOWESS trend line is locally weighted scatterplot smooth.)—Continued

but the locally smoothed values (indicative of central tendency) do not vary substantially over the range of four orders of magnitude in SSC. RSD is even more constant with respect to mean filtered volume, thus indicating no increase in PN variability in samples requiring low filter volumes because of high SSC.

Obtaining representative subsamples for preparation of filter pads is another factor that might affect PN variability. Subsamples can be extracted by drawing from a churn splitter or by pouring through a cone splitter. Some variability among replicate PN samples might be a result of using a separate subsample for each filter. The sampling protocol for the synopticfield study did not specify a method for subsample extraction, so there is no certainty that separate subsamples were used for each filter. If replicate PN samples were filtered from the same subsample, variability estimated from replicate standard deviations and RSD values might be low. The greatest impact would be for samples with SSC greater than $1,000 \mathrm{mg} / \mathrm{L}$, the upper limit for extraction of representative subsamples from a churn splitter (U.S. Geological Survey, variously dated, section 5.1.1). In this study, subsamples for all waters with SSC greater than 3,000 mg/L were extracted using a cone splitter, so these subsamples are more likely to be representative. Only 13 of 896 replicate sets had SSCs in the $1,000-3,000 \mathrm{mg} / \mathrm{L}$ range, for which representative subsamples might be questionable. An additional 42 samples had no reported SSC, but the $\mathrm{PN}$ concentrations in these samples were less than the range of PN measured in high SSC samples. It appears that sampling protocols were appropriate for obtaining representative subsamples, although deviation from standard protocols might be one explanation for large RSDs in some of the replicate sets. Other field procedures might also have contributed to large RSDs. For example, imprecise measurement of the volume of water passed through the filter could lead to large differences 

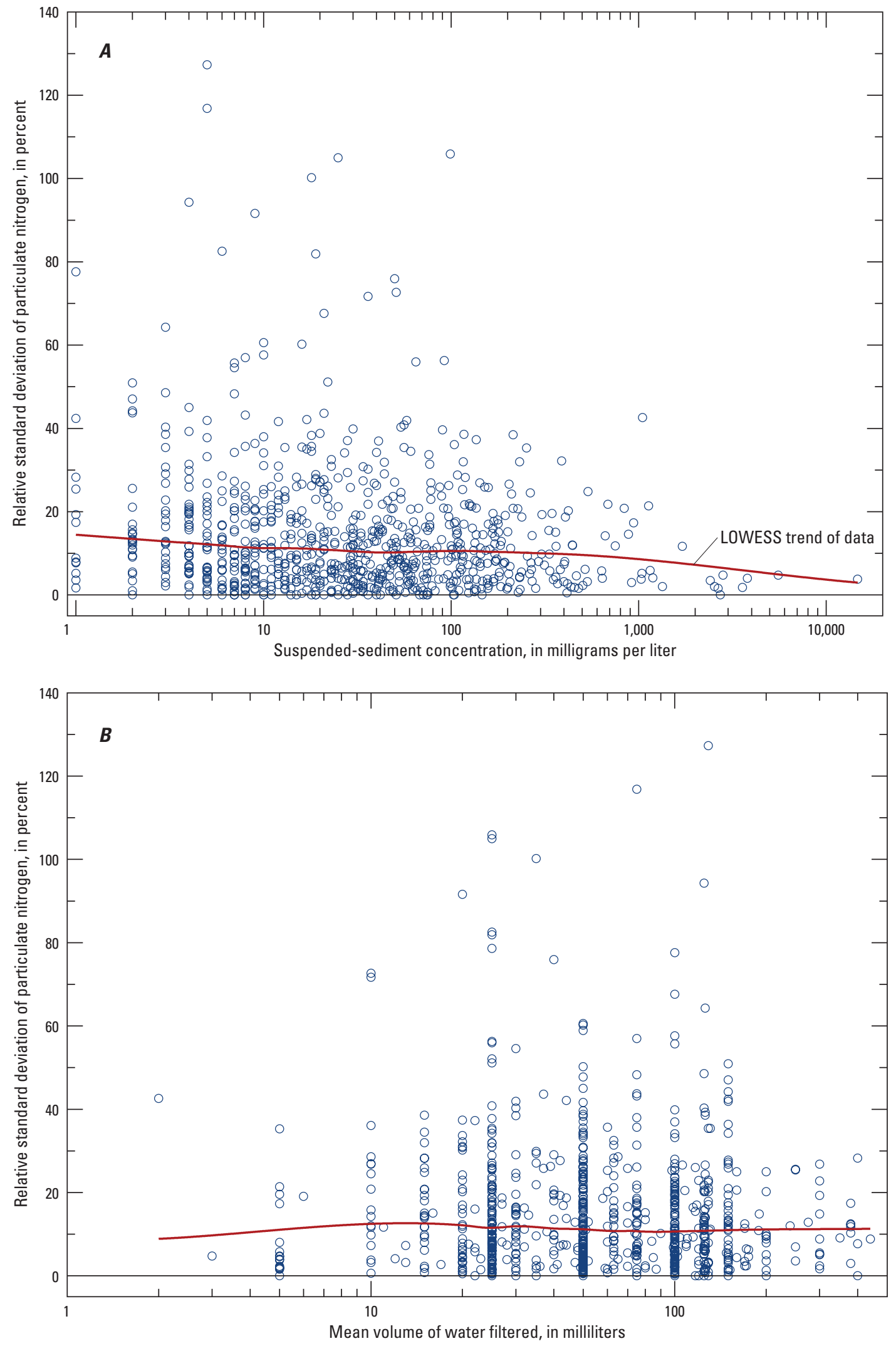

Figure 4. Relations of $(A)$ suspended sediment concentration and $(B)$ water volume filtered to relative standard deviation of particulate-nitrogen measurements in replicate samples collected for the synopticfield study, 2009-10. 
in computed PN concentration in replicate samples. Overall, the field component of variability seems likely to be more important for PN than for other nitrogen species.

\section{Precision of Computed Total Nitrogen}

The precision of computed total nitrogen, whether the sum of DN and PN (TN-C) or TKN and $\mathrm{NO}_{\mathrm{X}}$ (TN-K), depends on the proportion of each analyte in the sample. For TN-C, if $\mathrm{DN}$ is predominant, precision would be affected primarily by the variability of DN (estimated in this study at 2.3 percent of the analytical result). If $\mathrm{PN}$ is predominant, precision would decrease because of the higher variability of PN results (about 13 percent). If the concentrations of $\mathrm{DN}$ and $\mathrm{PN}$ in a sample are approximately equal, the expected variability of $\mathrm{TN}-\mathrm{C}$ would be intermediate, perhaps about 7-8 percent. For the 905 samples from the synoptic-field study, the average proportion of PN to TN was about 14 percent, and only 34 samples had proportions of PN to TN greater than 50 percent. Therefore, for most samples variability of TN-C likely approximates that of DN.

Similarly for $\mathrm{TN}-\mathrm{K}$, precision depends on the proportion of TKN and $\mathrm{NO}_{x}$. Based on table 5, for samples with concentrations greater than 1 , if $\mathrm{NO}_{\mathrm{X}}$ is predominant, the expected variability of TN-K would be smaller (RSD about 2 percent) than if TKN is predominant (RSD about 8 percent). The mean proportion of TKN to TN-K in 212 synoptic-field samples was about 40 percent, and 70 samples had proportions greater than 50 percent. In this case, the higher variability of TKN has a substantial effect on the precision of total nitrogen computed as TN-K. Thus the expected variability of TN-K is intermediate between 2 and 8 percent. For most samples, the precision of total nitrogen computed as TN-C would be better (lower variability) than the precision of TN-K.

\section{Assessment of Bias in Total-Nitrogen Methods}

Bias in measured TN-A and TN-K was computed by comparison to known TN in synthetic samples prepared for the laboratory experiment or in comparison to measured TN-C for samples collected during the synoptic-field study. Sources and mechanisms of bias were investigated to the extent possible for each of the analytical methods: alkaline persulfate digestion for TN-A and Kjeldahl digestion for TN-K.

\section{Assessment of Bias Determined by the Laboratory Experiment}

The nutrient-fortified, deionized-water matrix for the laboratory experiment contained nitrate $(2.0 \mathrm{mg} / \mathrm{L}$ as N) and glycine $(0.3 \mathrm{mg} / \mathrm{L}$ as $\mathrm{N})$ as the sole components of $\mathrm{DN}$. Standard reference materials provided uniform sources of
PN. Interpretation of results obtained for various additions of reference materials was more straightforward than would have been possible for complex environmental water matrices. Whereas the Buffalo River sediment and Montana soil reference materials were selected to approximate characteristics of suspended sediment in environmental river samples, the Green River Shale reference material, with its very high nitrogen and carbon content, was selected to challenge the alkaline-persulfate and Kjeldahl digestion methods.

In both methods, nitrogen bias was extremely negative for the samples amended with Green River Shale (tables A1 and $\mathrm{A} 2$ in the appendix), a clear indication that either the reagent concentration was insufficient to completely digest the PN or that some fraction of PN was refractory (resistant to digestion), even under the extreme conditions of heat and acidity of the Kjeldahl digestion. As expected, these results pointed out the limitations of both methods, but because Green River Shale represents nutrient-enrichment conditions that are not expected in environmental streams, these results were not included in further assessment of the bias in nitrogen analytical methods.

\section{Bias in the Alkaline-Persulfate and Kjeldahl Methods}

As stated earlier, reagent limitation caused by high concentrations of organic materials in sediment-laden samples was suspected as the primary mechanism for negative bias in TN-A. However, results from laboratory experiments with Buffalo River sediment and Montana soil (fig. 5) revealed negative bias in TN-A results, even at the lowest sediment concentrations tested (100 and $200 \mathrm{mg} / \mathrm{L}$, respectively). Note, however, that minimum sediment concentrations in the synthetic samples were substantially greater than many SSCs typically encountered at field sites. In general, the negative bias in TN-A was greater at higher sediment concentrations but to different degrees for each of the reference materials. Negative bias was smaller for samples prepared with Montana soil at all levels of sediment addition. For both reference materials, variability of results among sample replicates was small in the laboratory experiment.

In addition to the comparisons of TN-A at various sediment concentrations, $14 \mathrm{TN}-\mathrm{A}$ analyses were performed on the nutrient-fortified water with no sediment reference material added. The TN-A bias of these samples, listed in table A1 in the appendix, varied by $0.05 \mathrm{mg} / \mathrm{L}$ and averaged $0.00 \mathrm{mg} / \mathrm{L}$, indicating essentially no bias in the alkaline-persulfate method when no sediment is present.

TKN bias showed a less pronounced trend in relation to sediment concentration than did bias in TN-A (fig. 5). Bias in TKN ranged from about 5 percent negative to more than 10 percent positive, and in general, there was a wide range in results for each reference material at all sediment concentrations. Measured TKN values were positively biased for the Montana soil samples, but the LOWESS smooth for the Buffalo River sediment samples indicates a slight shift 


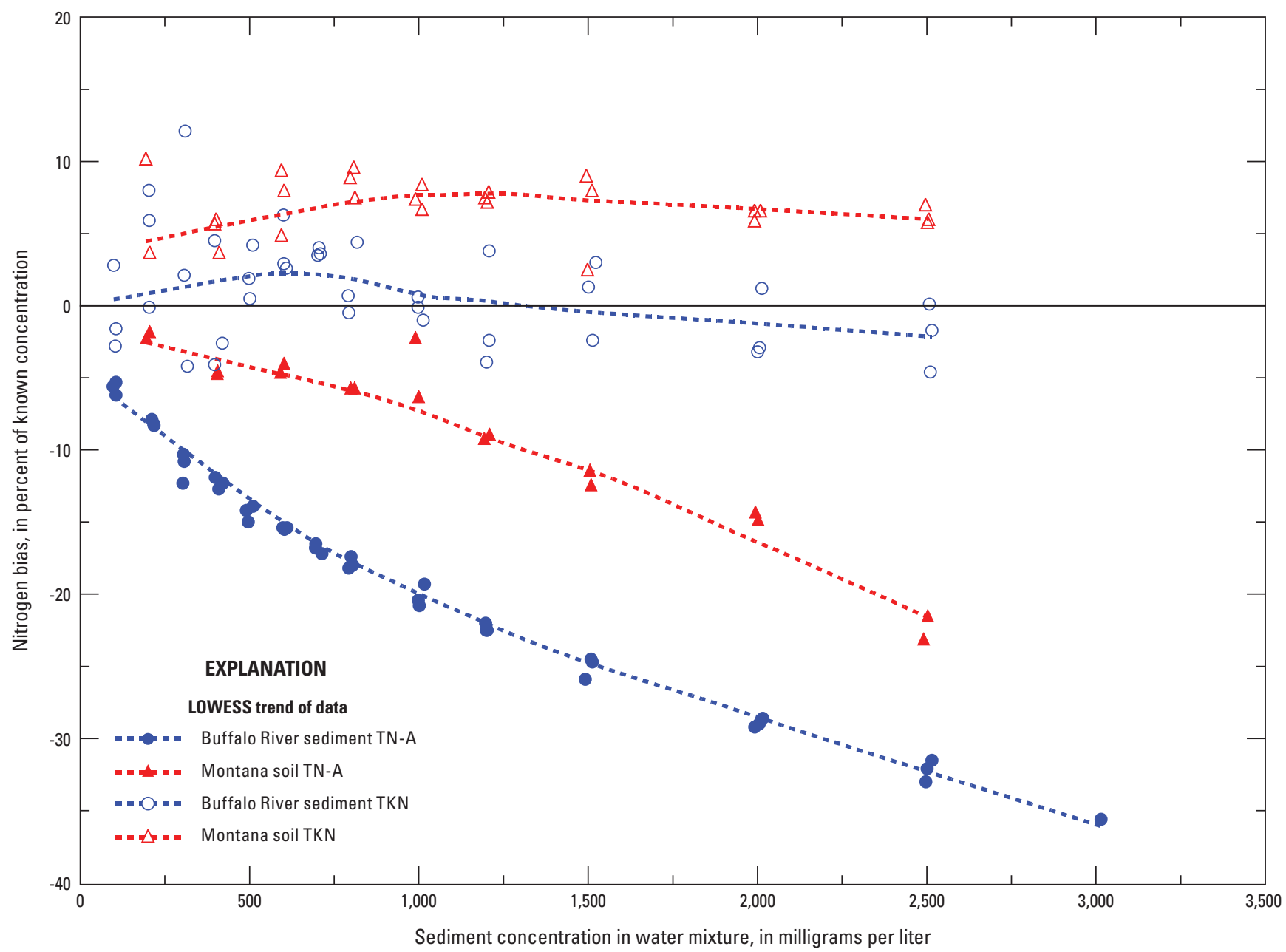

Figure 5. Bias in total nitrogen determined by alkaline-persulfate digestion (TN-A) and total Kjeldahl nitrogen (TKN) in laboratory tests using synthetic samples made from nutrient-fortified water and various concentrations of two reference materials: Buffalo River sediment or Montana soil.

from generally positive to negative bias in TKN for sediment concentrations above $1,000 \mathrm{mg} / \mathrm{L}$. This suggests that an abundance of sediment might affect the extent of nitrate reduction during Kjeldahl digestion, and that bias in TN results is likely to vary among field sites. The presence of negatively biased TKN may be the result of PN sequestration analogous to that observed for TN-A. In this regard, it is noteworthy that a small and variable fraction of $\mathrm{PN}$ resistant to Kjeldahl digestion in bed sediments from the tidal Potomac River and estuary was documented in an earlier USGS study (Simon and Kennedy, 1987). Simon and Kennedy recovered this sequestered PN fraction by dissolving post Kjeldahl-digestion particulate residues in hydrofluoric acid. In this study, HTCO analysis revealed a sequestered PN fraction in wet chemical-digest residues. Considering the near 100 percent recoveries of PN from reference sediments by HTCO (fig. 1), it is reasonable to assume that HTCO would be at least as effective as hydrofluoric acid dissolution with respect to releasing any PN fraction not recovered by commonly used wet-chemical digestion procedures.

\section{Source of Bias in the Alkaline-Persulfate Method}

The potential for preventing reagent limitation in the alkaline-persulfate method was tested by analyzing digests prepared with half the volume of aqueous, nutrient-fortified matrix and twice the volume of persulfate reagent. In relation to the standard alkaline-persulfate method, this modification doubled the persulfate concentration and halved the dissolved nitrogen concentration in the aqueous matrix. Results of TN-A analysis using this fortified-reagent test are shown in figure 6 . Over the range of sediment concentration tested, relative bias in TN-A was generally 10 percent smaller using the fortified reagent but was still negative, indicating that reagent limitation was not the predominant factor.

After alkaline-persulfate digestion reagent limitation was ruled out as the primary mechanism of low nitrogen recovery, additional testing was done to determine whether the bias in TN-A was caused by sequestration or suppression. Sequestration would occur if some of the nitrogen in the reference material was refractory (resistant to digestion by the persulfate 


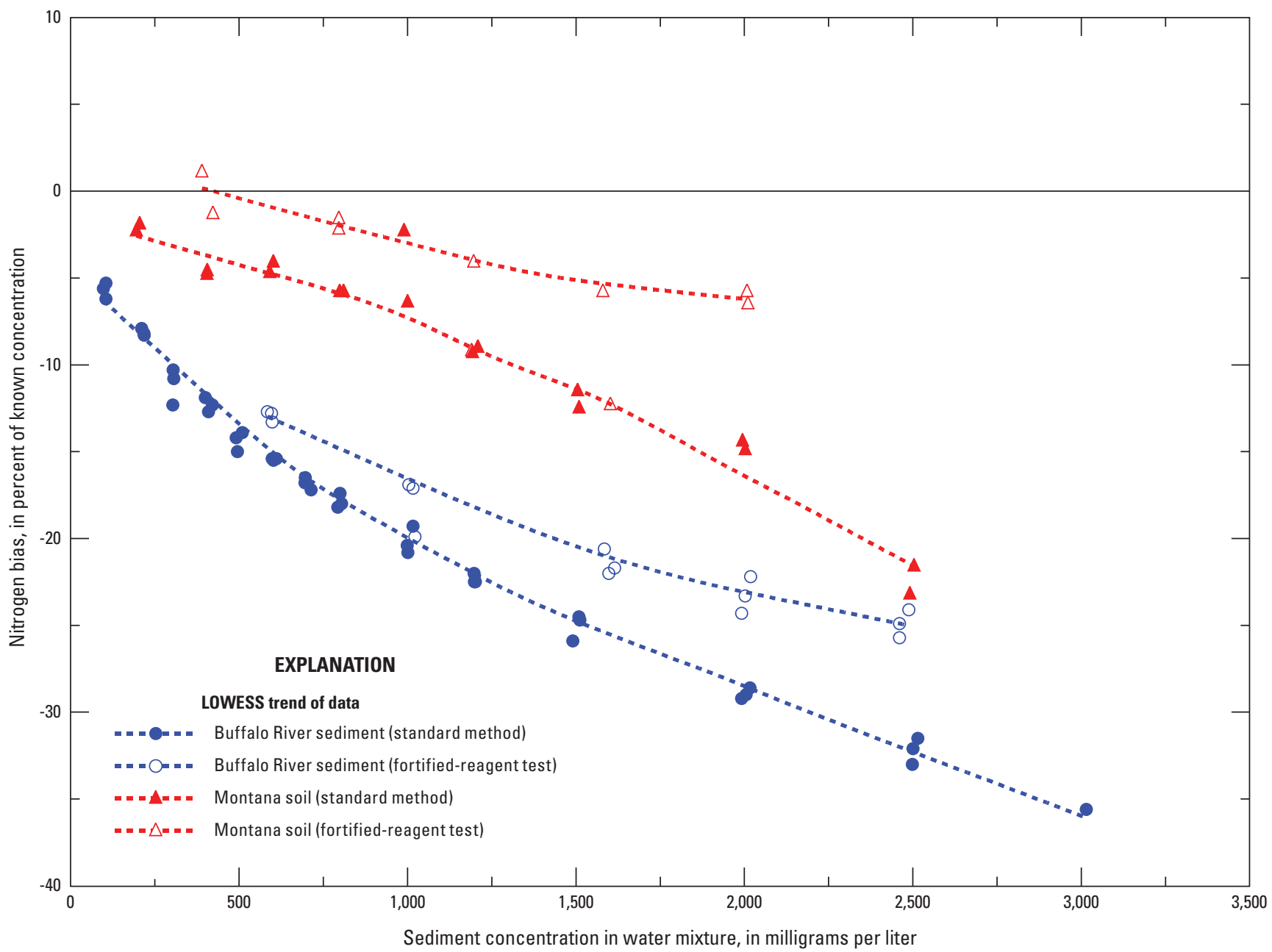

Figure 6. Comparison of bias in total-nitrogen concentration in synthetic samples analyzed using the standard alkalinepersulfate method and an alternate, fortified-reagent test using four times the reagent relative to the original sample volume.

reagent). Suppression would occur if some substance in the reference material interfered with colorimetric determination of nitrogen following digestion. The possibility of sequestration was qualitatively investigated using the HTCO PN method to analyze the residue remaining in alkaline-persulfate digest tubes. The PN measured in digest residues approximated the amount of nitrogen not recovered from alkalinepersulfate digests. This established, for the reference materials at least, that negative bias in TN-A resulted from sequestration rather than the suppression of a determinative step through reagent limitation.

To demonstrate the degree of sequestration occurring in the reference materials, apparent PN recovery by the alkalinepersulfate digestion method was computed as the ratio of the measured TN-A minus the known DN $(2.3 \mathrm{mg} / \mathrm{L})$ to the known PN concentration in the synthetic sample (known from reference material composition). The apparent PN recovery computation assumes dissolved nitrogen species were completely and accurately recovered, and reference material composition is known with accuracy. Apparent recoveries of PN averaged 58 percent for samples with Buffalo River sediment and 78 percent for samples with Montana soil (fig. 7). The apparent recovery of PN from Buffalo River sediment was substantially less overall and more variable for the smallest mass additions, perhaps because weighing errors or sample heterogeneity could cause proportionately greater differences among samples when mass additions are small. The lowest sediment concentrations in samples prepared with Montana soil samples were twice those of samples prepared with Buffalo River sediment, which might account for the comparatively better recoveries at smaller mass additions. The slight decrease in apparent PN recovery at SSCs above $2,000 \mathrm{mg} / \mathrm{L}$ suggests the onset of reagent limitation. These experimental results further support the hypothesis that some fraction of PN in each reference material is not recoverable by alkaline-persulfate digestion. The results also indicate that this fraction is relatively constant between sediment concentrations of 500 and $2,000 \mathrm{mg} / \mathrm{L}$, differs among reference materials, and likely would differ among environmental sediments. 


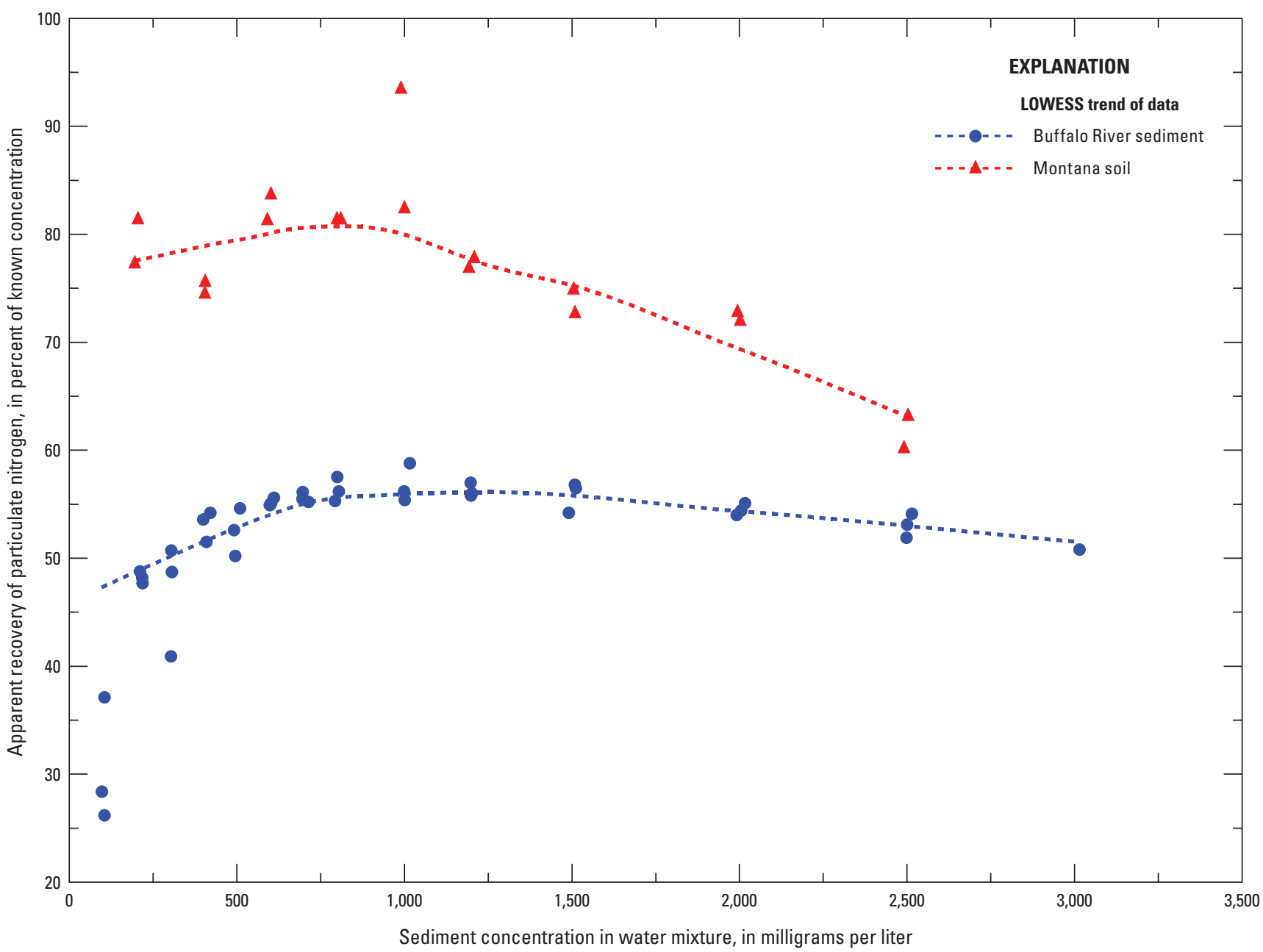

Figure 7. Apparent recovery of particulate nitrogen (assuming complete and accurate recovery of dissolved nitrogen species) in synthetic samples analyzed for total nitrogen by alkaline-persulfate digestion.

\section{Source of Bias in the Kjeldahl-Digestion Method}

The positive bias in TKN results for synthetic samples containing Montana soil indicates that nitrogen recovery is greater than 100 percent (fig. 5). This is also the case for most, but not all, of the samples containing Buffalo River sediment. Given that the nutrient-fortified matrix for these samples contained only glycine and nitrate, recoveries greater than 100 percent are likely the result of some nitrate being reduced to ammonia. Soil and crop scientists have used ${ }^{15} \mathrm{~N}$ isotopes to confirm this mechanism (Pace and others, 1982; Wyland and Jackson, 1994). For the laboratory experiment, the amount of nitrate reduction that occurred during Kjeldahl digestion was estimated by subtracting the known concentrations of organic nitrogen $(0.3 \mathrm{mg} / \mathrm{L}$ from glycine $)$ and PN from the measured TKN. Because no ammonia was present in the synthetic samples, the expected value of this difference - considered the apparent recovery of nitrate - would be zero if no nitrate reduction was occurring. The apparent recovery was normalized against the known concentration of nitrate $(2.0 \mathrm{mg} / \mathrm{L}$ as N) (fig. 8).
The discussion of nitrate interference in Standard Methods (American Public Health Association, 2012) indicates that nitrate interference in the Kjeldahl digestion is highly variable and can be positive or negative. This variability in TKN bias was reflected in the apparent recovery of nitrate in the synthetic samples amended with Buffalo River sediment, where the standard deviation of apparent-nitrate recovery at a given sediment concentration averaged 2.8 percent, and the trend of the recovery shifted from a positive to a negative bias. In contrast, for samples amended with Montana soil, the apparent recovery of nitrate was less variable (the standard deviation of apparent nitrate recovery at a given sediment concentration averaged 1.2 percent), consistently positive, and increased with greater additions of reference material. This pattern might be the result of increasing amounts of organic matter facilitating the reduction of more nitrate to ammonia. The greater variability for Buffalo River sediment might be related to higher actual TKN concentrations at each level of mass addition caused by the greater nitrogen content in this reference material ( 0.20 percent in comparison to 0.13 percent for Montana soil). Likewise, as sediment concentration increases, 


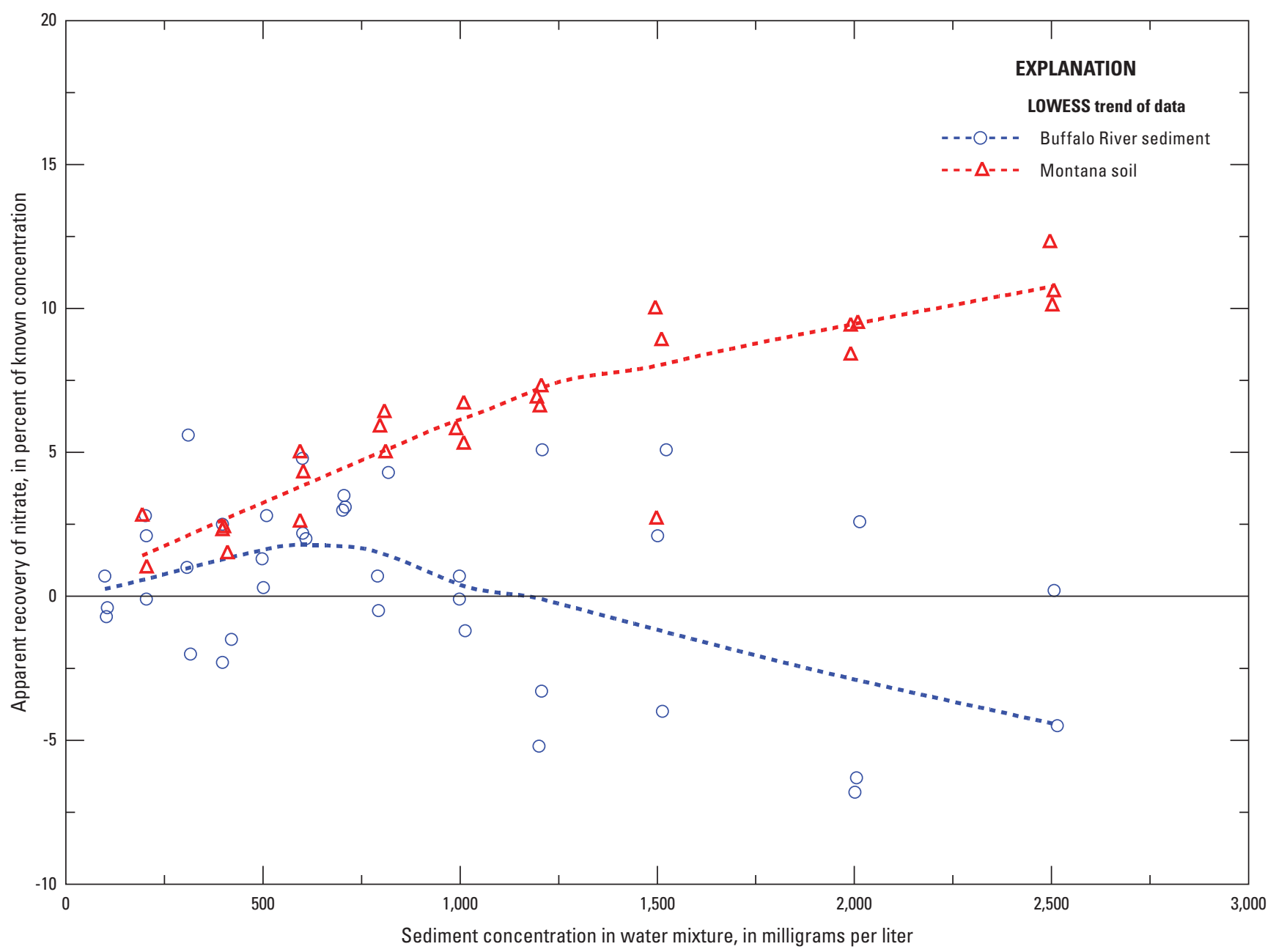

Figure 8. Apparent recovery of nitrate during Kjeldahl digestion (determined as the difference between measured and known concentrations of total Kjeldahl nitrogen in comparison to the known concentration of nitrate nitrogen) in synthetic samples amended with Buffalo River sediment or Montana soil.

the relative impact of sequestered PN would increase, possibly resulting in the observed minor shift from positive to negative bias for Buffalo River sediment at concentrations greater than $1,000 \mathrm{mg} / \mathrm{L}$ (figs. 5 and 8 ).

Several conclusions were drawn from the laboratory experiment:

- The Kjeldahl method is substantially less precise (more variable) than the alkaline-persulfate method.

- TN-A has a negative bias, primarily because some particulate nitrogen is refractory (resistant to alkalinepersulfate digestion), leading to sequestration of that nitrogen.

- The negative bias associated with the alkaline-persulfate method does not decrease substantially when sample volumes are halved and reagent volumes are doubled.

- For sediment-water mixtures typical of conditions in environmental streams, the Kjeldahl method could have a positive bias that may be a result of $\mathrm{NO}_{\mathrm{x}}$ reduction to ammonia or a negative bias as a result of particulate nitrogen that is refractory (resistant to digestion).

The overall conclusion from this laboratory experiment was that total-nitrogen concentrations determined by alkalinepersulfate digestion (in the presence of suspended sediment) or by summation of TKN plus $\mathrm{NO}_{\mathrm{x}}$ are likely to be biased. An alternative method is needed to accurately quantify the total concentration of nitrogen in whole-water samples.

\section{Assessment of Bias Determined by the Synoptic-Field Study}

The 905 geographically and temporally diverse samples from 77 sites in the synoptic-field study had TN-C concentrations ranging from 0.15 to about $26 \mathrm{mg} / \mathrm{L}$ as $\mathrm{N}$ and SSCs ranging from 1 to 14,700 mg/L (appendix table A4). All samples were analyzed for TN-A bias and 218 also were analyzed 

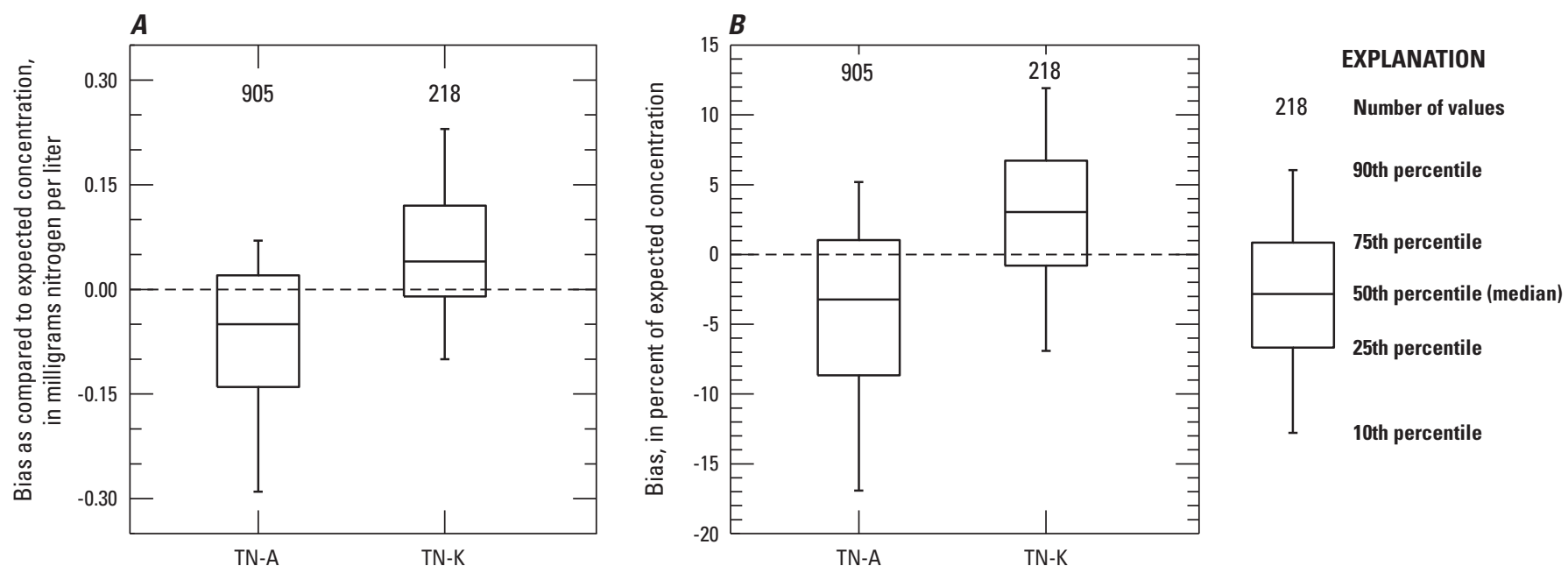

Figure 9. Distribution of $(A)$ bias and $(B)$ relative bias in total nitrogen determined by alkaline-persulfate digestion (TN-A) and as the sum of total Kjeldahl nitrogen and nitrate plus nitrite (TN-K) observed in the synoptic-field study, 2009-10.

for TN-K bias using the associated TN-C value as reference. Results from the synoptic-field study confirmed the biases revealed by previous laboratory and field data - generally negative biases in TN-A and positive biases in TN-K (fig. 9). The median TN-A bias was $-0.05 \mathrm{mg} / \mathrm{L}$ or -3.2 percent. The median TN-K bias was $0.04 \mathrm{mg} / \mathrm{L}$ or 3.1 percent.

\section{Bias in the Alkaline-Persulfate-Digestion Method}

Negative bias in TN-A was observed in the laboratoryexperiment results at the lowest SSCs evaluated $(100 \mathrm{mg} / \mathrm{L})$. The majority of samples (74 percent) in the synoptic-field study had SSCs less than $100 \mathrm{mg} / \mathrm{L}$, ranging down to $1 \mathrm{mg} / \mathrm{L}$. The negative bias appeared to be present over the entire range of observed SSCs (figs. $10 A$ and $10 B$ ).

These data were categorized into six ranges of SSC to evaluate the distribution of bias in each concentration range (fig. 11). In all six cases, the median TN-A bias was less than zero, suggesting that some degree of negative bias existed in the presence of even the lowest SSCs. In fact, the distribution of the relative bias of samples having SSCs from 1 to $10 \mathrm{mg} / \mathrm{L}$ was nearly as negative as the distribution of samples having SSCs from 101 to $750 \mathrm{mg} / \mathrm{L}$ (fig. 11B). Because the bias is present across the entire range of SSC, these results support the laboratory-experiment conclusion that negative bias is primarily from sequestration rather than digest-reagent limitation. It also demonstrates that on average, TN-A results are negatively biased even for samples with low SSCs.

Though still present, the pattern of increasingly negative TN-A bias with respect to SSC was not as well-defined in the synoptic-field study data as that for the laboratory experiment. Given the differences inherent between a controlled laboratory experiment and a field-sampling effort conducted at a national scale, an increase in variability around the general trend of the relation was not unexpected. One potential source of variability may have been related to the heterogeneity of sediments in the field samples. Not only would the composition of those sediments vary from site to site based on the soil and streambed characteristics of the contributing watershed, but they would also vary from sample to sample based on the dynamic nature of the erosional and sediment-transport processes that delivered the sediment to the stream for a given sample. However, a comprehensive determination of the sedimentary characteristics of each sample was outside the scope of this study.

With the exception of samples having SSCs greater than $750 \mathrm{mg} / \mathrm{L}$, the TN-A relative biases for the other five concentration ranges were similar (fig. 11B), with an overall median value of -3.2 percent. The abrupt expansion of negative bias in TN-A for samples with SSCs greater than $750 \mathrm{mg} / \mathrm{L}$ (fig. 11) indicates that another mechanism introducing bias, such as reagent limitation, might have operated at these higher SSCs.

Though in general the TN-A bias is more negative at SSCs greater than $750 \mathrm{mg} / \mathrm{L}$ (figs. $10 A$ and 11), there are still instances of only moderately negative and even positive TN-A bias at these higher SSCs. Likewise, there are instances of exceptionally negative TN-A bias at lower SSCs. These may suggest a dependence on the sediment composition or size distribution as well as concentration. Sediments with greater amounts of nitrogen bound to the particles $(\mathrm{PN})$ would be more prone to negative TN-A bias than sediments with low $\mathrm{PN}$, even if the overall SSC is held constant. This concept is illustrated when the TN-A biases in the synoptic-field study data are compared with PN concentration (fig. 10C) and suggests that SSC serves as a useful, though imperfect, predictor of $\mathrm{PN}$ in a given sample.

Locally weighted regressions (Helsel and Hirsch, 2002) were used to compare the trend of TN-A bias observed in the synoptic-field data with that of the earlier laboratory experiment (fig. 12). Although variability in the field data 

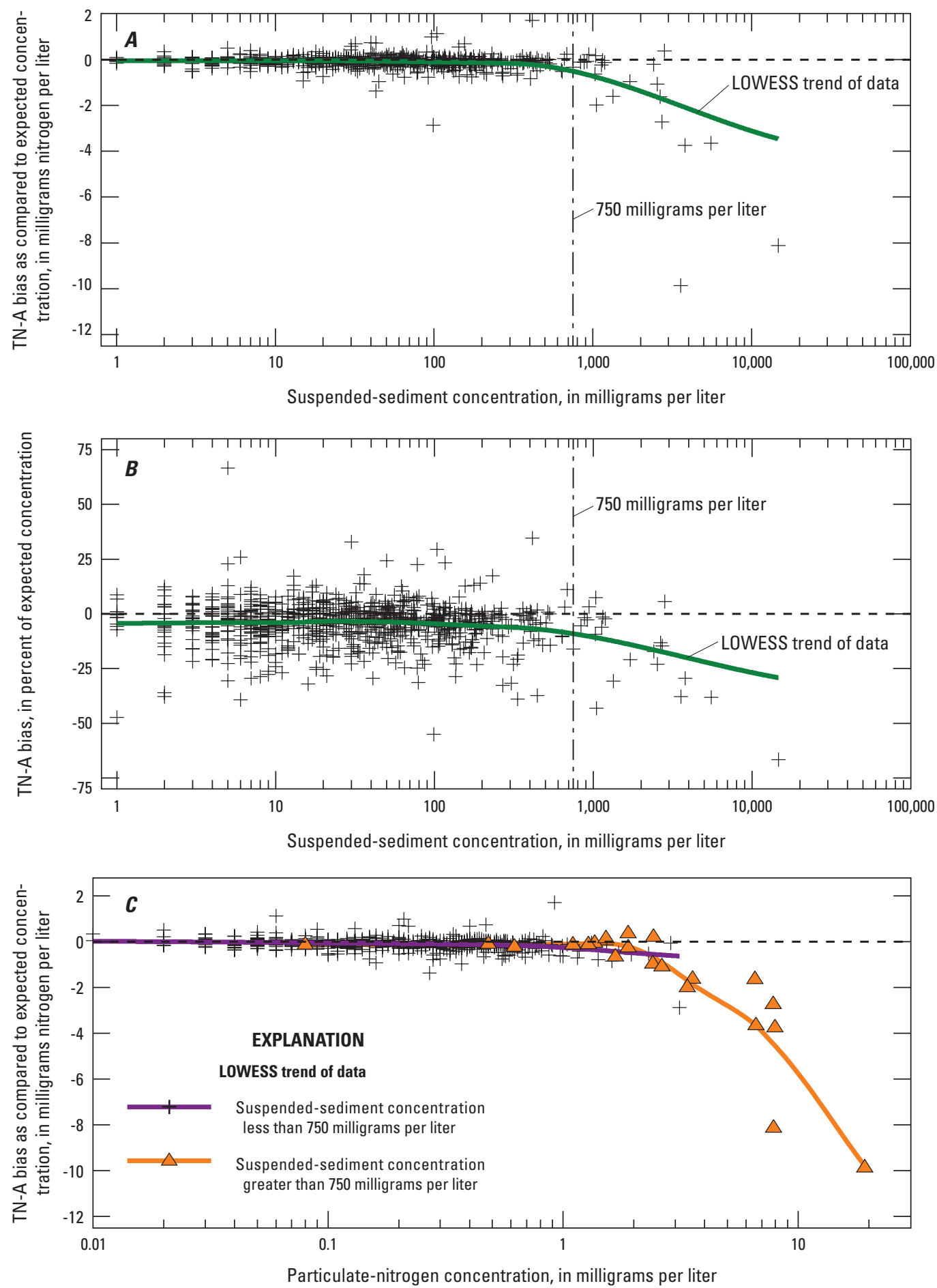

Figure 10. Relations of bias in total nitrogen determined by alkaline-persulfate digestion (TN-A) in samples collected as part of the synoptic-field study to $(A$ and $B)$ suspended-sediment concentration and $(C)$ particulate-nitrogen concentration 


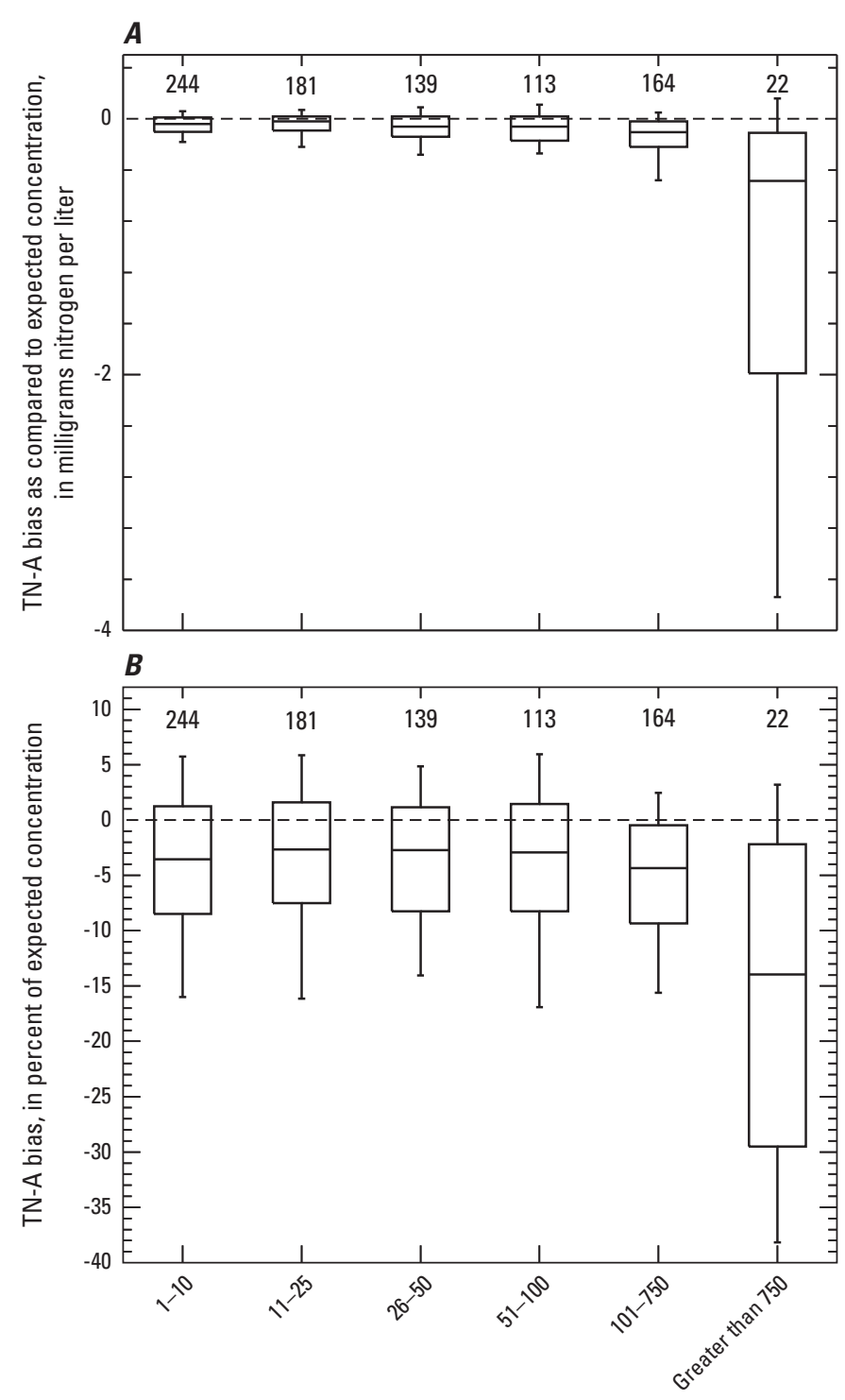

Suspended-sediment concentration range, in milligrams per liter

EXPLANATION

181 Number of values

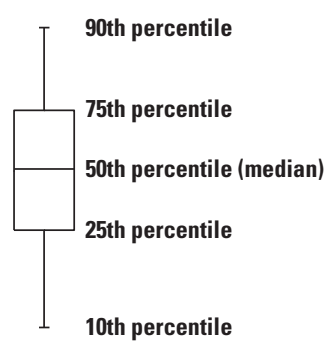

Figure 11. Distribution of $(A)$ bias and $(B)$ relative bias in total nitrogen determined by alkaline-persulfate digestion (TN-A) in samples collected for the synoptic-field study and categorized by suspended-sediment concentration range. was greater than in laboratory experiment data, the LOWESS trends are similar. This indicates that the findings of the laboratory experiment bracket the observations from the field- the reference materials used for the laboratory experiment were found to be representative of the broad range of sediments, and PN content thereof, included in suspended-sediment samples from the nationwide field study. At lower SSCs, the regression line for the synoptic-field data indicated a constant negative relative bias that averages approximately 5 percent of the actual concentration (figs. $11 B$ and $12 B$ ), confirming the presence of negative bias in samples with low SSC. At SSCs above $750 \mathrm{mg} / \mathrm{L}$, relative bias became more negative in the synoptic-field data as well as in the synthetic samples (figs. 7 , $11 B$, and $12 B$ ).

\section{Spatial Patterns of Bias in Total Nitrogen Determined by Alkaline-Persulfate Digestion}

Results from the synoptic-field study were evaluated for evidence of regional or land-use effects on the magnitude of TN-A bias. Mean bias was calculated for 57 sites with TN-A results from at least 10 samples (fig. 13). Though certain areas, particularly sites draining the central Plains and intermountain basins, tended to have more negative bias (fig. 13A), these distinctions became less clear when viewed as relative bias (fig. $13 B$ ). This suggests that the negative bias in TN-A was largest when nitrogen concentrations generally were higher, rather than simply as a function of geographical location.

Four basin characteristics were used to comparatively assess the mean relative bias in TN-A for selected sites in the synoptic-field study. These characteristics were computed using areal overlay techniques of Nakagaki and others (in press) for all sites having at least 10 measurements of relative bias in TN-A (appendix table A5). The versions of the digital basin delineations used in the analyses were current as of September 21, 2012. Results from the laboratory experiment indicated that TN-A bias might be affected by sediment type. Therefore, the percentage of silt and clay in the topsoil of the watershed (Natural Resources Conservation Service, 2010) was evaluated as a proxy for sediment type. However, the distribution of the mean relative bias in TN-A for the 57 synoptic-field sites did not covary with the amount of silt and clay in topsoil (fig. 14A). The amount of PN present in the soil might also affect the magnitude of TN-A bias, and the predominant land use (Fry and others, 2011) was presumed to represent soils that may be enriched with nitrogen. Though agriculturally dominated basins were expected to have high nitrogen enrichment, only basins dominated by rangeland seemed to have stronger negative relative bias in TN-A (fig. 14B). Given the small number of sites in each landuse category, this finding is not conclusive. Predominant ecoregions (Omernik, 1987) and physiographic divisions (Fenneman and Johnson, 1946) provided general but potentially relevant information about the watersheds. Comparisons of relative bias in TN-A among ecoregions were not 

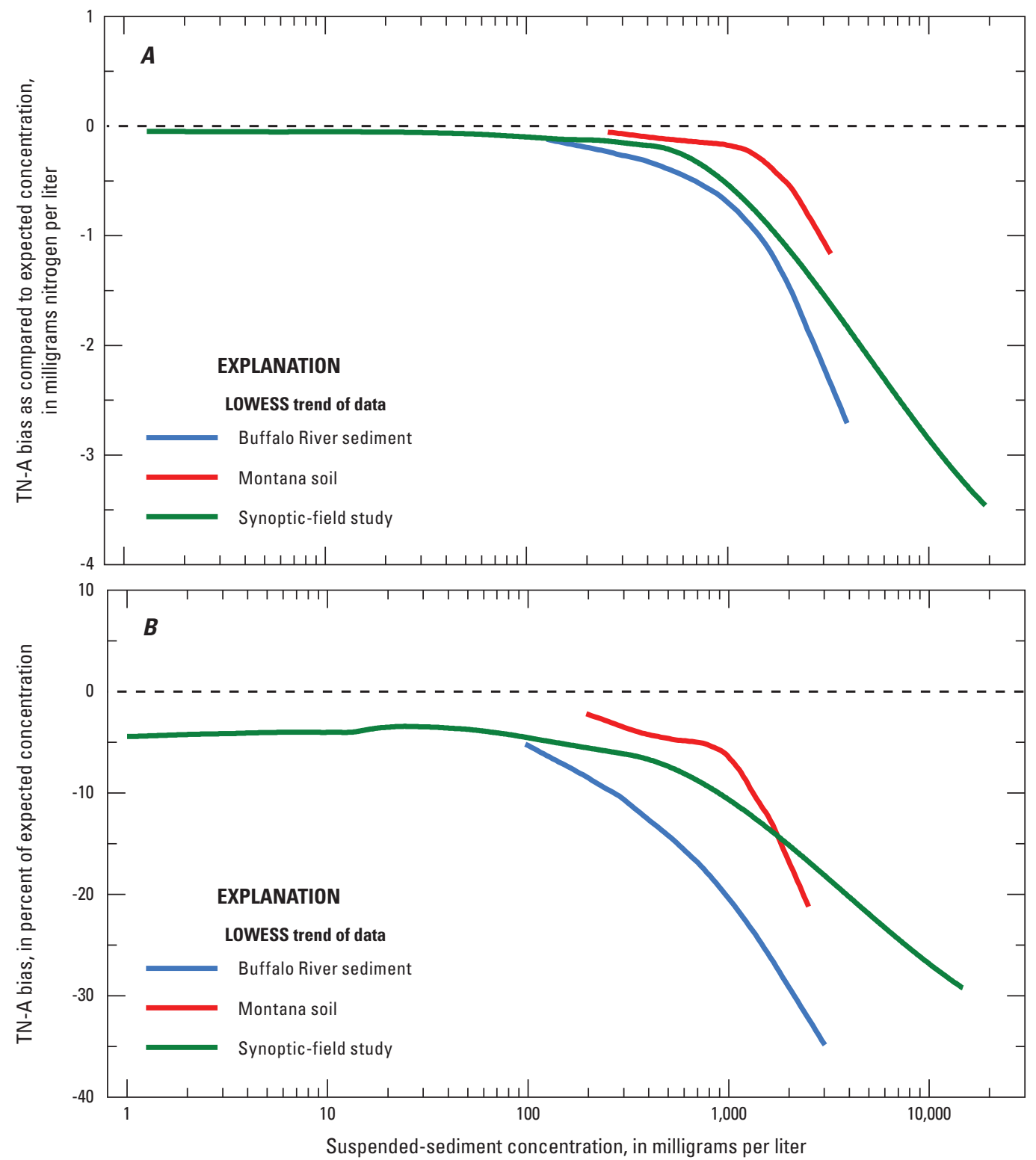

Figure 12. General trends (as represented by locally weighted regression) of (A) bias and (B) relative bias in total nitrogen determined by alkaline-persulfate digestion (TN-A) in sediment and soil samples from the laboratory experiment and synoptic-field study.

meaningful because of the small number of sites in each ecoregion grouping. Of the six physiographic divisions represented among the sites, the Interior Plains, Intermontane Plateaus, and Pacific Mountain System may be regions with somewhat more negative TN-A bias (fig. 14C). As with land use, however, these differences may also be a coincidental artifact of the small number of sites in most groups. Given that the overall distribution of relative bias in TN-A did not vary greatly until SSC was greater than $750 \mathrm{mg} / \mathrm{L}$ (fig. 11B), a lack of relation between mean relative bias and these basin characteristics is not unexpected.

\section{Bias in the Kjeldahl Method}

Bias was evaluated in TN-K concentrations measured in the synoptic-field samples, with a particular focus on the effect of $\mathrm{NO}_{\mathrm{X}}$ on that bias. For the 218 samples from 19 sites having sufficient data for TN-K bias analysis, the median TN-K bias was $0.04 \mathrm{mg} / \mathrm{L}$ as $\mathrm{N}$ (fig. $9 A$ ) or 3.1 percent (fig. $9 B$ ).

The synoptic-field study supported the findings of the laboratory experiment in which the Kjeldahl-digestion method was shown generally to produce a positive bias in the presence of elevated $\mathrm{NO}_{\mathrm{x}}$ (fig. 15). Whereas $\mathrm{NO}_{\mathrm{x}}$ concentration in the laboratory samples did not vary, the synoptic-field study 

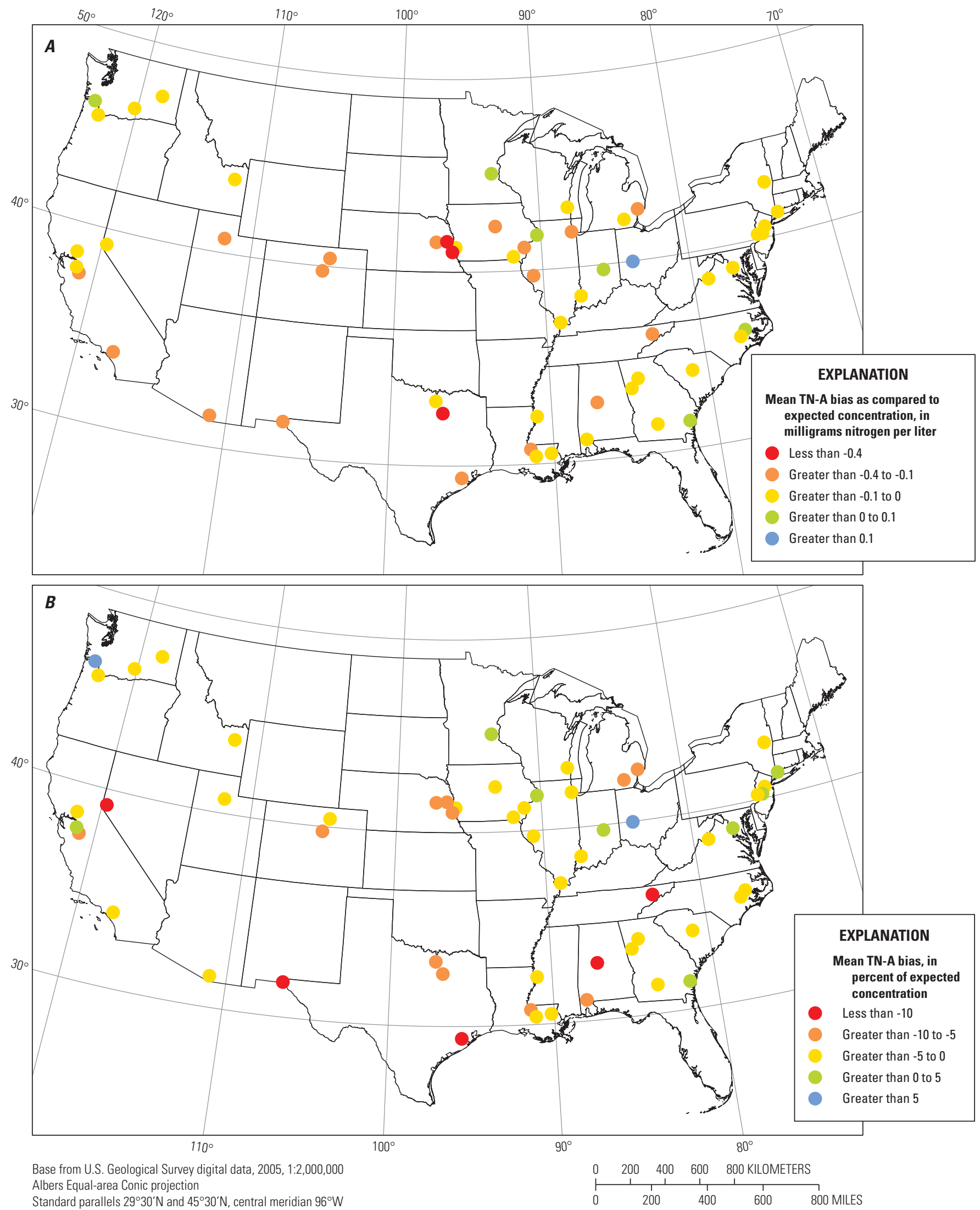

Standard parallels $29^{\circ} 30^{\prime} \mathrm{N}$ and $45^{\circ} 30^{\circ} \mathrm{N}$, central meridian $96^{\circ} \mathrm{W}$ North American Datum of 1983

Figure 13. (A) Mean bias and $(B)$ mean relative bias in total nitrogen determined by alkaline-persulfate digestion (TN-A) for sampling sites with 10 or more measurements, synoptic-field study, 2009-10. 

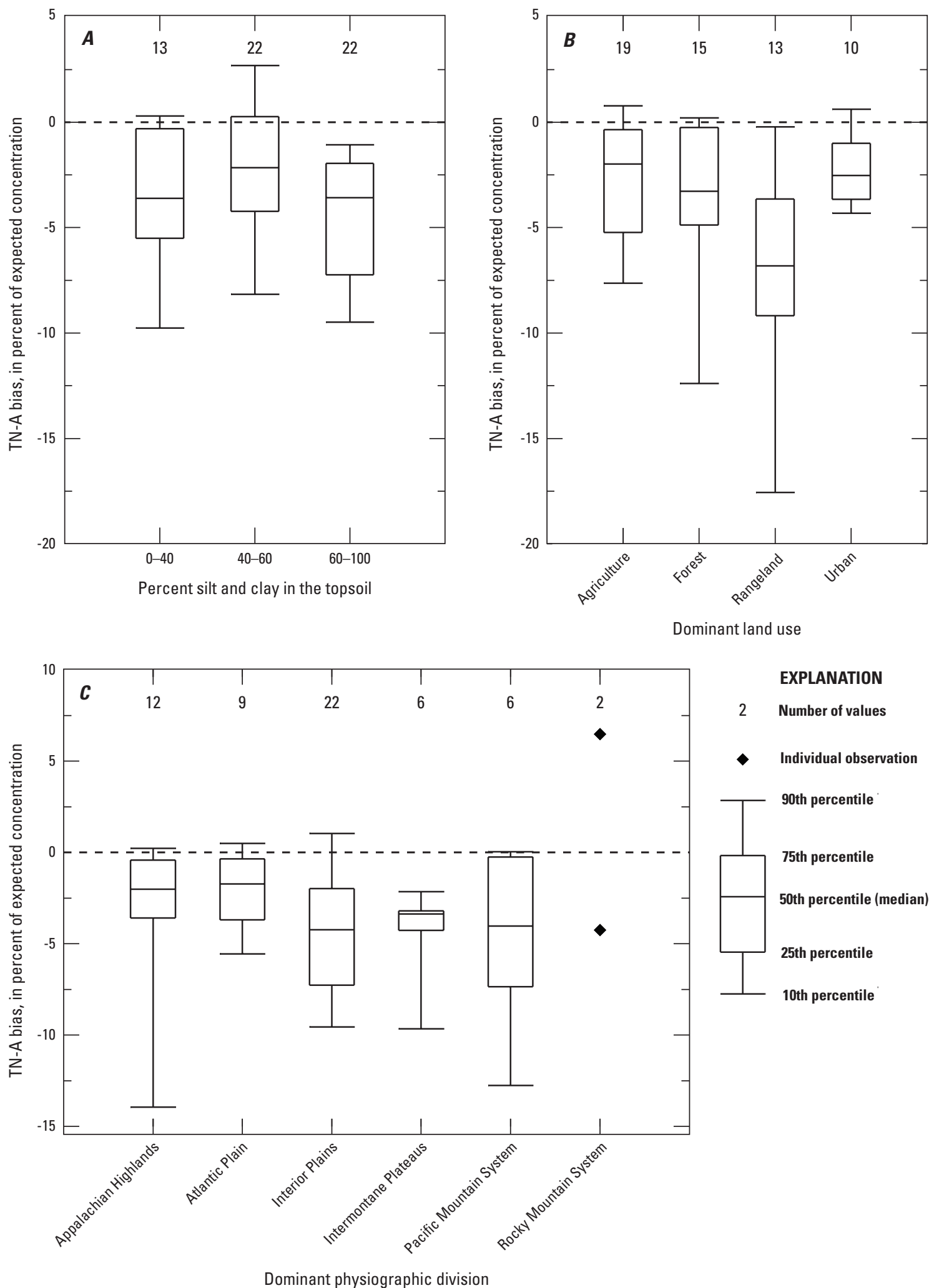

Figure 14. Comparisons of relative bias in total nitrogen determined by alkaline-persulfate digestion (TN-A) to drainage-basin characteristics for selected sampling sites in synoptic-field study, 2009-10, for $(A)$ percent silt and clay in topsoil, $(B)$ dominant land use, and $(C)$ dominant physiographic division. 


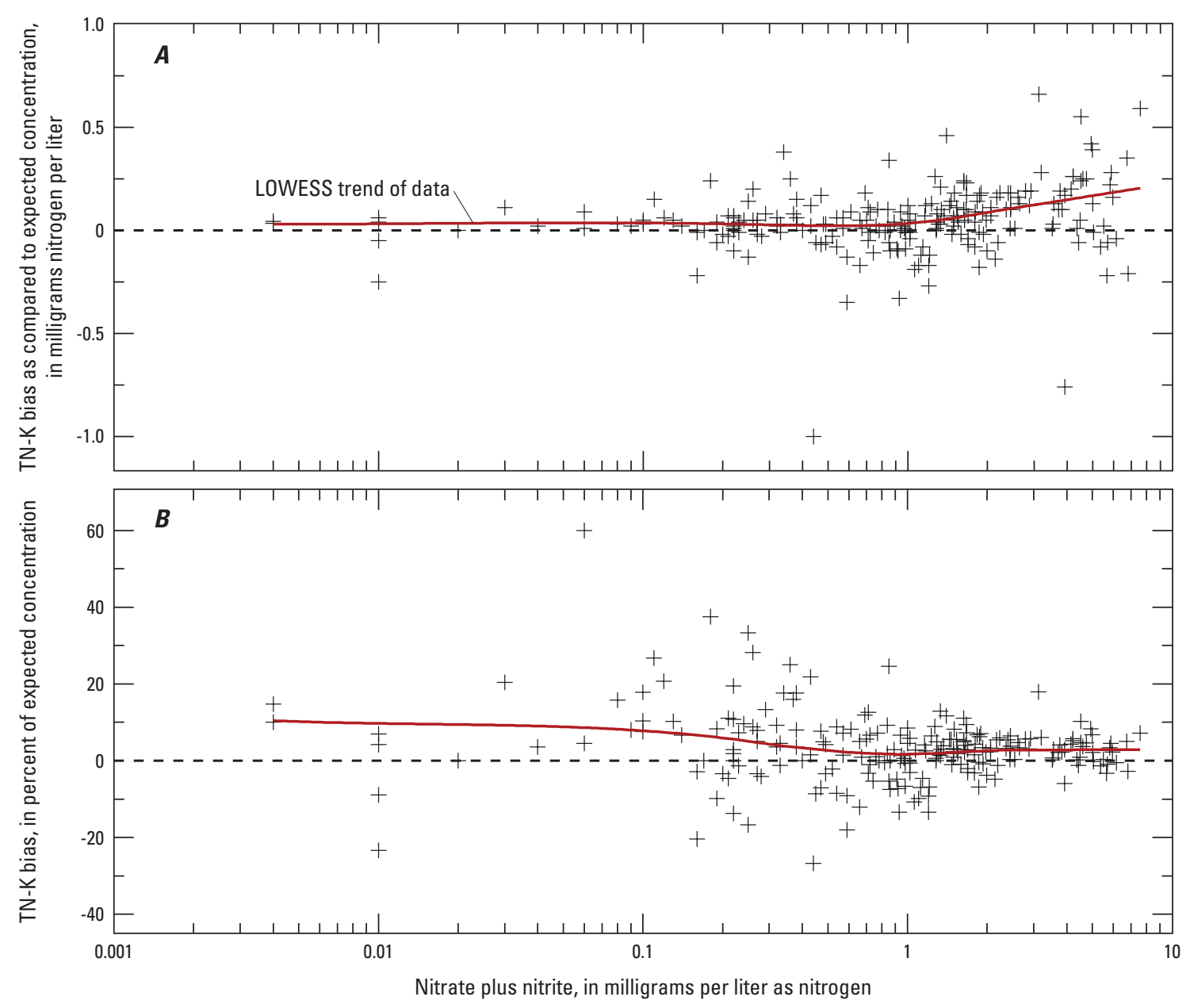

Figure 15. Relations of bias and relative bias in total nitrogen computed as the sum of total Kjeldahl nitrogen and nitrate plus nitrite (TN-K) to nitrate plus nitrite concentration in samples collected as part of the synopticfield study, 2009-10.

included samples with $\mathrm{NO}_{\mathrm{X}}$ ranging from less than the reporting level of 0.016 to about $7.6 \mathrm{mg} / \mathrm{L}$ as $\mathrm{N}$ (appendix table $\mathrm{A} 4$ ). In the same manner that bias in TN-A was further assessed by ranges of SSC, the bias in TN-K was grouped by ranges of $\mathrm{NO}_{\mathrm{X}}$ concentration (fig. 16). TN-K bias showed a slightly positive relation to concentration of $\mathrm{NO}_{\mathrm{X}}$ (figs. $15 A$ and $16 A$ ) with variability increasing proportionally with $\mathrm{NO}_{\mathrm{x}}$. Though it was generally positive, the relative bias in $\mathrm{TN}-\mathrm{K}$ was more variable at lower concentrations of $\mathrm{NO}_{\mathrm{X}}$ and tended to become less positive with increasing $\mathrm{NO}_{\mathrm{X}}$ (figs. 9, 15B, and $16 B)$. The occurrence of positive TN-K bias in the presence of elevated $\mathrm{NO}_{\mathrm{X}}$ was expected given the documented potential for $\mathrm{NO}_{\mathrm{X}}$ to be reduced to ammonia during Kjeldahl digestion. This would produce an artificially high TKN value, thereby causing a positive bias in the computation of TN-K (American Public Health Association, 2012; Patton and Truitt, 2000; Patton and Kryskalla, 2003, p. 23-25 and fig. 8). This mechanism of bias may be causing the positive bias in TN-K seen in the results from the 2009-10 synoptic-field study. However, a conclusively upward trend in relative bias as concentrations of $\mathrm{NO}_{\mathrm{X}}$ increased was not apparent.

Four major conclusions were drawn from the synopticfield study:

- The patterns in bias observed in field samples are supported by findings of the laboratory experiment.

- Negative bias in TN-A results was present regardless of SSC, but it becomes more likely and larger at SSCs greater than $750 \mathrm{mg} / \mathrm{L}$.

- Relative bias in TN-A does not appear to be explained by variations in the characteristics selected to describe the upstream drainage basin.

- Though variable, positive bias in TN-K is typical in the presence of elevated $\mathrm{NO}_{\mathrm{X}}$. 


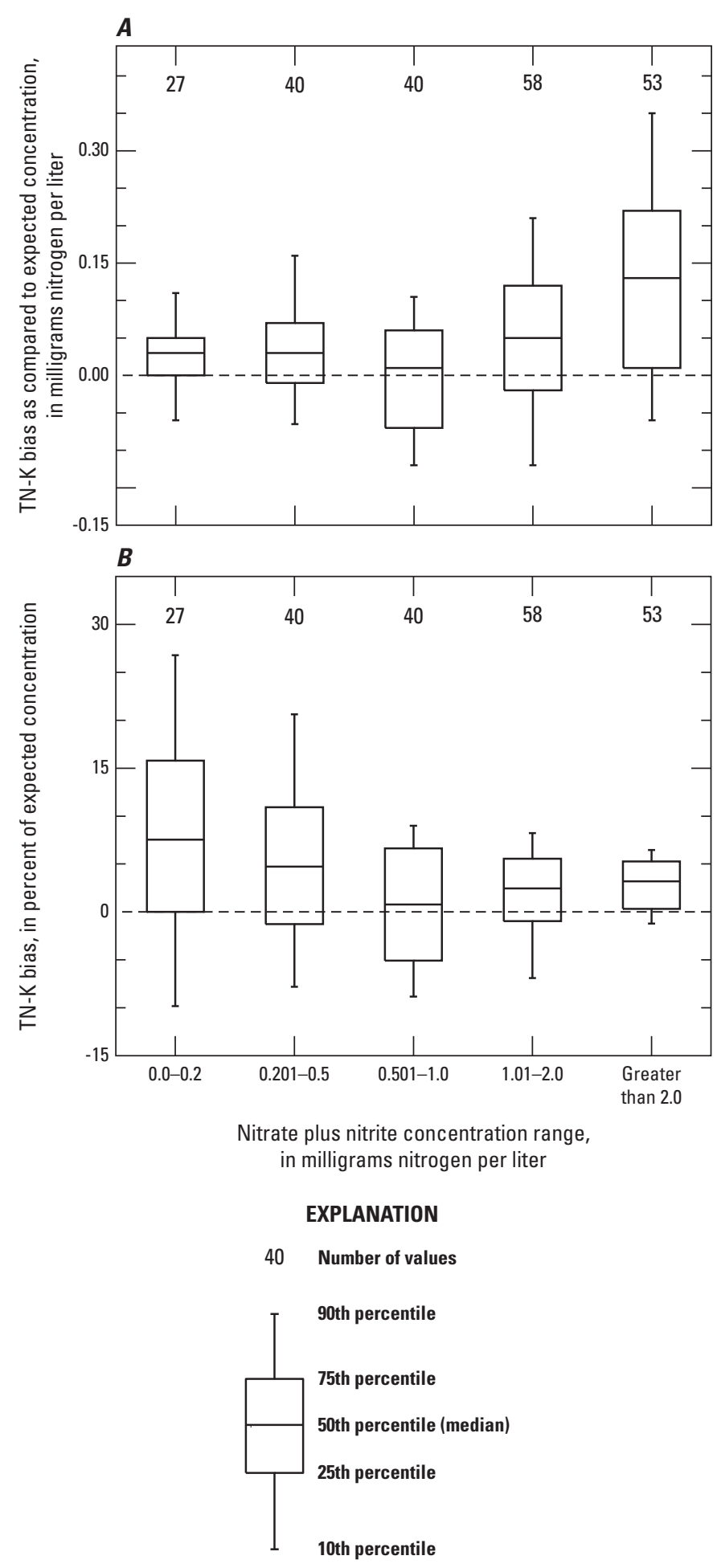

Figure 16. Distribution of $(A)$ bias and $(B)$ relative bias in total nitrogen determined as the sum of total Kjeldahl nitrogen and nitrate plus nitrite (TN-K) observed in samples collected for the synoptic-field study, 2009-10, among ranges of nitrate plus nitrite concentration. Values outside the $10^{\text {th }}$ and $90^{\text {th }}$ percentiles are not shown.

\section{Evaluation of Alternatives for Determining Total Nitrogen}

Data from the laboratory experiment and the synopticfield study yielded estimates of precision for TN concentrations determined using methods TN-A, TN-K, and TN-C as well as bias in the TN-A and TN-K methods. The evaluation of the advantages and disadvantages of each approach for determining TN is less straightforward, but of equal importance. Such an evaluation must consider the impacts on field and laboratory procedures, including costs, and on data interpretation, including any discontinuity in the historical record that might result from a methodological change. These impacts, which will likely vary considerably for different sampling programs, will challenge USGS scientists trying to establish uniform standards for TN sample collection and analytical determinations. In the paragraphs that follow, three alternatives were considered for programs currently collecting TN-A or TN-K data:

1. Replace TN-A with TN-C,

2. Replace TN-K with TN-C, and

3. Addition of TN-C, perhaps only temporarily, to existing analyses of TN-A or TN-K.

\section{Factors Considered}

Each analytical approach was evaluated in relation to: (1) anticipated precision and bias of analytical results, (2) impacts on existing field and laboratory practices, and (3) impacts on data interpretation and the historical record. There might be additional considerations for a particular sampling program, but in this report the focus is on the above criteria that are common concerns across all programs.

\section{Anticipated Precision and Bias}

Precision and bias of TN values estimated by each approach will differ by individual sample and protocols in place for sampling programs. A generalized graphical summary of expected precision and bias for $\mathrm{TN}$ values resulting from the TN-A, TN-K, and TN-C approaches is shown in figure 17. More detailed discussions of precision and bias for each approach are included in earlier sections of this report. Figure 17 characterizes the precision of calculated values of TN (TN-C and TN-K) as a range associated with the precision of each analyte used in the calculation. However, the actual precision is dependent on the relative proportions of the nitrogen analytes within the sample. This study found that $\mathrm{DN}$ is typically predominant; therefore, $\mathrm{TN}-\mathrm{A}$ precision is probably similar to that of the more precise DN method. In contrast, TKN often composed 40 percent or more of TN-K, 


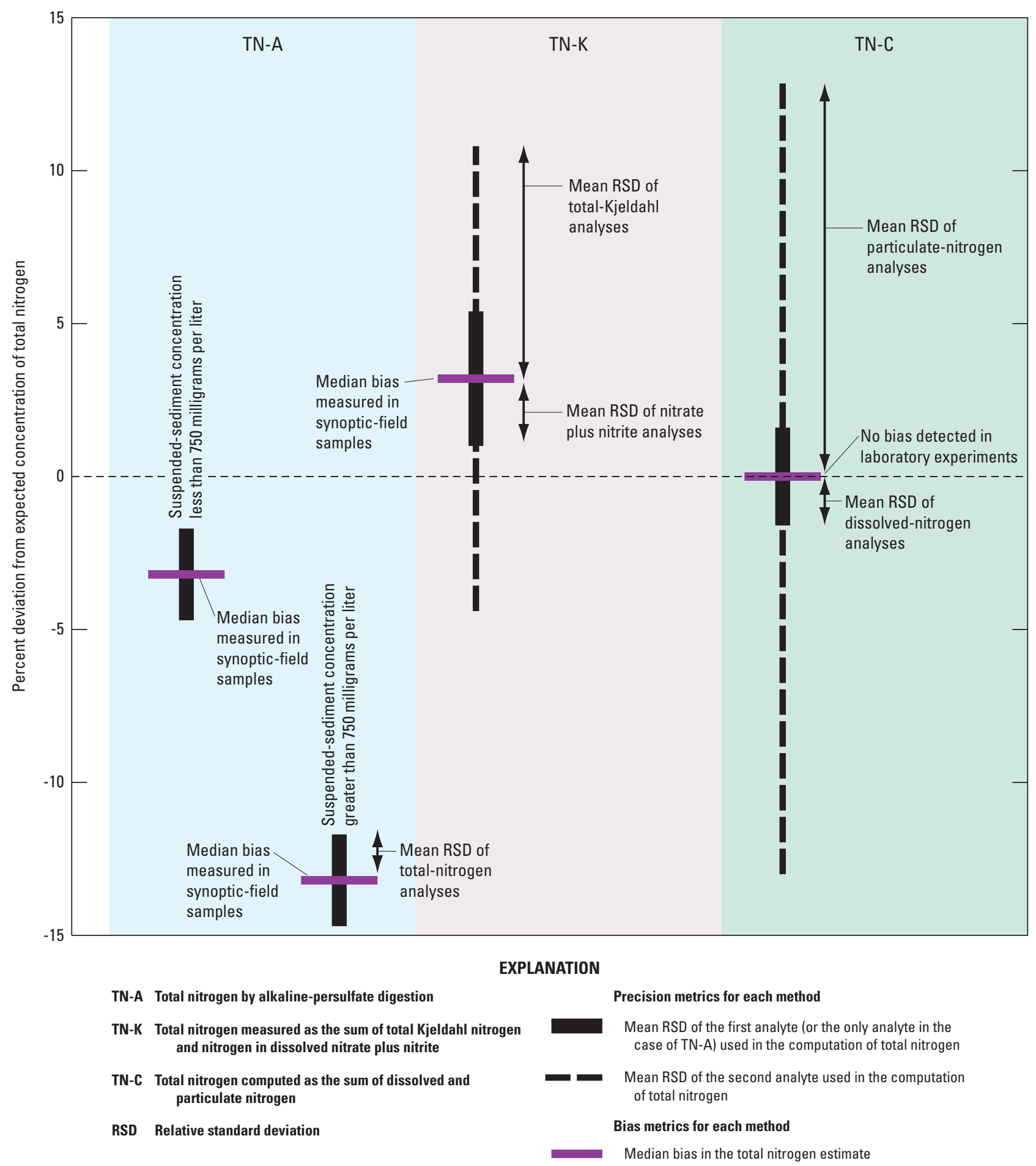

Figure 17. A graphical depiction of the relative effect of precision and bias on three methods for determining total nitrogen (ranges in precision for computational methods reflect the corresponding precision of the methods for individual analytes used in the computation). 
suggesting that TN-K precision is likely intermediate between the precision of its two analytes. Within this report, precision is characterized for both high and low concentrations of total nitrogen. Furthermore, with reference to figure 17, negative bias in TN-A seems to be dependent on SSC, particularly at concentrations greater than $750 \mathrm{mg} / \mathrm{L}$, and positive bias in $\mathrm{TN}-\mathrm{K}$ results is most pronounced for samples containing high concentrations of $\mathrm{NO}_{\mathrm{X}}$.

\section{Impact on Field and Laboratory Procedures}

Each alternative considered for determining TN includes the addition of TN-C. Collection of whole-water (unfiltered) samples is fundamental to USGS sampling programs, most of which currently submit whole water for analysis by the alkaline-persulfate or Kjeldahl methods and filtered water for analysis of dissolved species such as $\mathrm{NO}_{\mathrm{X}}$ and ammonia. Adding analysis of DN in these filtered-water samples is unlikely to affect sample-collection costs, and likewise, replacing one whole-water method with another is unlikely to affect sampleanalysis costs. On the other hand, processing whole-water aliquots to prepare filter pads for PN analyses would increase sampling costs because preparing filter pads for PN analyses is an additional step in sample processing. Some sampling programs might incur additional costs of buying PN filtration equipment. Time spent cleaning equipment and preparing PN filter pads would vary; experience gained during the 2009-10 synoptic-field study suggests that 0.5 person-hour per site is typical, but the additional time could be considerably more for sediment-laden samples.

With the inclusion of TN-C in each approach, laboratory impacts are primarily dependent on potential sample loads for DN and PN determinations. Analytical capacities at the NWQL for DN are likely sufficient to handle increased analytical loads that might result from any of the three approaches under discussion. However, additional equipment and personnel might be required to satisfy the increased demand that might come with the inclusion of HTCO analyses for nutrient samples.

Because the suite of nutrients collected varies by sampling program, the relative financial impacts of these approaches would differ among sampling programs. The relative cost comparison provided in this report pertains to a typical nutrient analysis consisting of nitrate, nitrite, ammonia, phosphorus, orthophosphate, and one of the methods of obtaining total nitrogen. The cost impact for programs utilizing different sets of constituents will vary accordingly. Estimated costs are based on published USGS NWQL pricing for 2012. For the alternatives considered in the report, the prices are presented as a percentage of the cost of the existing analyses (TN-A or TN-K).

\section{Impact on Data Analyses}

There are many uses of total nitrogen data-ambient stream assessment, trend analyses, and load calculation to name a few-and limitations of any of the approaches considered might affect these interpretations. Reduced precision of data would correspondingly reduce the power of statistical analyses and might affect conclusions reached from those analyses. Biased data might affect the stream classification or loading rates from a given stream. Additional complications in selecting a TN assessment approach might arise if a regulatory program does not recognize a particular method. Analyticalmethod changes implemented during ongoing multiyear studies might introduce artificial trends in TN as a result of methodological rather than environmental changes. Alternatively, maintaining use of a biased method might suggest artificial trends if the mechanism driving the bias (such as the presence of sediment) varies from year to year. Spatial analyses could be affected if different sampling programs opted for different approaches to TN assessment. Furthermore, an individual data point could be used for many different purposes, and an approach that satisfies one set of data-quality objectives might be detrimental to another.

\section{Replace TN-A with TN-C}

As described in earlier sections of this report, TN-A results from laboratory experiments with reference materials and from synoptic field samples of environmental water confirmed that some fractions of PN resist oxidation to nitrate by TN-A methods, thereby introducing a negative bias. The median bias in TN-A measured from the synoptic-field study was $-0.05 \mathrm{mg} / \mathrm{L}$ or -3.2 percent. By comparison, recoveries of PN by HTCO elemental-analysis methods appeared unbiased based on close agreement with reference values across a range of sediment masses. Alkaline-persulfate digestion of filtered samples generally is accepted as the reference method for determining DN, and other studies have found unbiased recovery of DN by alkaline-persulfate digestion (Vandenbruwane and others, 2007, section 3.1 and references cited therein). Because TN-C is the sum of PN and DN, TN-C estimates of TN are expected to be unbiased (table 6). The effect of removing negative bias in TN data by using the TN-C approach should be more marked in waters with SSC greater than about $750 \mathrm{mg} / \mathrm{L}$, and in such regimes might have a greater impact on conclusions (such as nitrogen loads or stream classifications) drawn from those data. Although bias also was detected in samples with low SSCs, it was of lesser magnitude.

However, there are several drawbacks to replacing TN-A with the TN-C method (table 6). More field time would be spent preparing samples for analysis, and analytical costs for a typical nutrient sample would increase by approximately 56 percent. The PN method is less precise than the TN-A method. The imprecision of PN transfers loss of precision to the TN-C estimate in increasing magnitude as the relative contribution of PN to TN increases, though the majority of 
samples in the synoptic-field study was dominated by DN rather than PN. Although samples with very high SSC (and a correspondingly large contribution of $\mathrm{PN}$ ) are atypical of the majority in most USGS sampling programs, they also may represent conditions during which a large proportion of nitrogen transport by a stream occurs.

The replacement of one analytical method with another would complicate time-series analyses that span that replacement. The expected removal of bias from subsequent samples may lead to step trends that are explained by the method change rather than an environmental condition. Also, the potential for reduced precision may prevent otherwise significant trends from being detected. It is important to note, though, that trends computed from existing and future TN-A data also may be affected by negative bias that covaries with SSC.

\section{Replace TN-K with TN-C}

As described in previous studies and earlier sections of this report, the presence of high levels of $\mathrm{NO}_{\mathrm{X}}$ in a sample may result in the conversion of some $\mathrm{NO}_{\mathrm{X}}$ to ammonia during the digestion process, thereby introducing a positive bias into TN-K estimates. For the synoptic-field study, the median TN-K bias was $0.04 \mathrm{mg} / \mathrm{L}$ or 3.1 percent. Alternatively, the TN-C method does not appear to be affected by this bias. Therefore, sampling programs currently utilizing the TN-K method may consider its replacement with the TN-C method.

The primary benefit of this approach is the apparent removal of positive bias from TN-K estimates (table 6). The removal of bias may lead to improved assessments of nitrogen load or stream classifications.

However, a switch from TN-K to TN-C methods may affect time-series analysis such as trend detection (table 6). Because the TN-K method has been in use for a longer period of time than the TN-A method, there are likely to be more historical data associated with the TN-K method, and a switch to TN-C from TN-K would have a greater impact than a switch to TN-C from TN-A. This alternative likely would not be adopted by regulatory programs that might continue to require the use of TN-K for stream assessment. Operationally, this approach would require added field processing time and an analytical cost increase of around 50 percent for a typical nutrient sample.

\section{Add TN-C Analyses to Existing Analyses of TN-A or TN-K}

Whereas the replacement of TN-A and TN-K analyses with TN-C is expected to alleviate bias in TN estimates, it also introduces difficulties, including loss of precision, and USGS lacks a period of concurrent measurements from which the impact of a method change can be evaluated. Therefore, an approach that adds TN-C analyses to TN-A or TN-K analyses is worthy of consideration. Operationally, this approach would follow the basic techniques of the synoptic field study in which two estimates of TN would be quantified using different methods. For the majority of samples, the TN-A or TN-K data would be considered the primary estimate of TN. However, the magnitude of bias in those data would be known from comparison to the TN-C value. If that bias exceeded a predefined threshold, the TN-C value would be considered the primary estimate of TN. In the context of figure 17, this approach recognizes that for most samples, the amount of bias in TN-A or TN-K may be bracketed by imprecision of the TN-C method. Only when bias exceeds the threshold would the alternative TN-C analyses be preferred for their lack of bias.

This approach would maintain consistency with historical data for the majority of samples and would prevent a minority of samples from being affected by excessive bias (table 6). This approach would likely improve the accuracy of load estimates or stream classifications in sediment-laden streams. The overlapping data could be used to evaluate the impact that bias or a method change might have on temporal trends. In addition, the data set for comparing TN-A with TN-C would expand both in sample number and spatial coverage, thereby allowing for future evaluations of this approach.

The primary drawback to this approach is that it presents the largest burden on sampling programs (table 6). Sampling crews would be required to collect whole-water, filtered-water, and filter-pad samples. The additional analytical costs associated with DN and PN would not be offset by the removal of TN-A or TN-K, thereby resulting in an 83- or 78-percent cost increase for typical nutrient samples that currently use TN-A or TN-K, respectively. Data management also would be complicated by the inclusion of two estimates for the same constituent and the resulting determination of which value should be considered primary for use in interpretive analyses. In addition to these operational burdens, the continued use of TN-A or TN-K for the majority of samples may perpetuate the presence of bias of lesser magnitude in subsequent datasets.

\section{Summary}

The characterization of total-nitrogen concentrations is an important component of many surface-water-quality programs. This report focuses on the challenges and limitations of three widely used methods for routine determination of total nitrogen in water: (1) total nitrogen (TN-A) derived from the alkaline-persulfate digestion of whole-water samples (2) total nitrogen (TN-K) calculated as the sum of total Kjeldahl nitrogen $(\mathrm{TKN})$ and dissolved nitrate plus nitrite $\left(\mathrm{NO}_{\mathrm{X}}\right)$; and (3) total nitrogen (TN-C) calculated as the sum of dissolved nitrogen $(\mathrm{DN})$ and particulate nitrogen $(\mathrm{PN})$ (See Glossary for total-nitrogen definitions). Specifically, it explores how suspended-sediment and nitrate concentrations affect the performance of these methods, both in synthetic media-laboratory-formulated, nutrient-fortified deionized-water suspensions of reference materials (river sediment and soil) — and in 
Table 6. Summary of advantages and disadvantages associated with three alternatives to current U.S. Geological Survey practices for determining total-nitrogen concentration in surface-water samples.

[TN-A, total nitrogen measured by alkaline-persulfate digestion of a whole-water sample; TN-C, total nitrogen computed as the sum of dissolved and particulate nitrogen; DN, dissolved nitrogen measured by alkaline-persulfate digestion of a filtered sample; $\mathrm{NO}_{\mathrm{x}}$, nitrogen in dissolved nitrate plus nitrite; $\mathrm{TKN}$, total Kjeldahl nitrogen; TN-K, total nitrogen measured as the sum of $\mathrm{TKN}$ and $\mathrm{NO}_{\mathrm{x}}$.]

\begin{tabular}{|c|c|c|}
\hline Advantages & Disadvantages & $\begin{array}{l}\text { Analytical cost change } \\
\text { relative to existing practice }\end{array}$ \\
\hline \multicolumn{3}{|c|}{ Alternative: Replace TN-A with TN-C } \\
\hline \multicolumn{3}{|c|}{ Alternative: Replace TN-K with TN-C } \\
\hline $\begin{array}{l}\text { +Positive TN-K bias removed from subsequent data, } \\
\text { especially in } \mathrm{NO}_{\mathrm{x}} \text {-enriched streams } \\
\text { +Improvement of subsequent load estimates or } \\
\text { stream classifications }\end{array}$ & $\begin{array}{l}\text {-Potential for less precision than TN-K (though } \\
\text { most samples will approach the precision of } \\
\text { DN, which exceeds the precision of TN-K, } \\
\text { see fig. 17) } \\
\text {-Method replacement may introduce an artificial } \\
\text { step-trend into time-series analysis of histori- } \\
\text { cal data } \\
\text {-Regulatory programs may stipulate the con- } \\
\text { tinued use of TKN, thereby adding to the } \\
\text { financial burden of this alternative } \\
\text {-Increased field processing time } \\
\text { (0.5 person-hours) }\end{array}$ & 50 percent increase. \\
\hline \multicolumn{3}{|c|}{ Alternative: Add TN-C to existing TN-A or TN-K analyses } \\
\hline $\begin{array}{l}\text { +Negative TN-A bias removed from subsequent data } \\
\text { in which bias exceeds a predefined threshold } \\
\text { +Maintains the higher precision of the TN-A method } \\
\text { for the majority of samples } \\
\text { +Improvement of subsequent load estimates in } \\
\text { sediment-laden streams } \\
\text { + Maintains consistency with historical data } \\
\text { +Expands the number of samples and sites by which } \\
\text { negative TN-A bias can be determined } \\
+ \text { Transition period allows for the impact of bias and } \\
\text { method changes on interpretations of the data to } \\
\text { be characterized }\end{array}$ & $\begin{array}{l}\text {-Of the three alternatives, this presents the } \\
\text { largest financial burden specific to sample } \\
\text { collection and analysis } \\
\text {-Increased field processing time } \\
(0.5 \text { person-hours }) \\
\text {-The inclusion of a second estimate of TN } \\
\text { would complicate data management and may } \\
\text { confuse data users } \\
\text {-Bias of lesser magnitude will be maintained for } \\
\text { the majority of subsequent samples }\end{array}$ & $\begin{array}{l}83 \text { percent increase for } \mathrm{TN}-\mathrm{A} \\
\text { analyses. } \\
78 \text { percent increase for } \mathrm{TN}-\mathrm{K} \\
\text { analyses. }\end{array}$ \\
\hline
\end{tabular}

${ }^{1}$ Estimated relative to the cost of a typical nutrient sample that includes total nitrogen, ammonia, nitrate, nitrite, orthophosphate, and phosphorus analyses.

a set of over 900 geographically and compositionally diverse environmental water samples collected from 77 U.S. Geological Survey (USGS) stream-sampling sites during June 2009 through September 2010.

A comparison of 10 large-volume water samples in 2005 suggested a negative bias in TN-A when compared to TN-C data. A series of follow-up studies were undertaken to identify the cause and extent of this negative bias and to explore some remedial measures. The first was a 2007 laboratory experiment designed to identify a connection between suspended sediment and negative bias in TN-A, to explore potential remedies, and to detect positive bias in TN-K in the presence of elevated $\mathrm{NO}_{\mathrm{X}}$. A synoptic-field study was undertaken in 2009-10 to evaluate the efficacy of using $\mathrm{TN}-\mathrm{C}$ as a more accurate alternative to TN-A for water samples. In addition, data were collected to address whether variability in analytical results for $\mathrm{PN}$ might introduce excessive error in TN-C, thereby creating one problem while alleviating another.

Analytical variability was assessed primarily using data from the synoptic field study. At low concentrations, the 
analytical methods tended to perform comparably with respect to variability. At higher concentrations, the relative variability was about 2 percent for methods that utilized colorimetric determinations of $\mathrm{NO}_{\mathrm{x}}$ following alkaline-persulfate digestion (TN-A, DN), but about 13 percent for PN, and 7.6 percent for the Kjeldahl-digestion (TKN) method. Comparisons of PN precision with the corresponding filtered-water volume indicated that filtered volume had little relation to relative variability over a range of four orders of magnitude in PN.

$\mathrm{TN}-\mathrm{A}$ precision outperformed that of the analytes in the TN-K and TN-C methods. The computed concentrations of total nitrogen with the TN-K or TN-C methods are less precise than direct measurements of TN-A. However, the loss of precision depends on the relative amount of TKN or PN in the total nitrogen computation. For the synoptic field study, PN (having a precision of 13 percent) constituted on average only 14 percent of TN-C, suggesting that the precision of the TN-C method approaches that of DN (2.3 percent). On the other hand, TKN (having a precision of 7.6 percent) constituted on average 40 percent of TN-K, suggesting that the imprecision of the Kjeldahl digestion likely affects the TN-K estimates adversely. For most samples, the precision of total nitrogen computed as TN-C would be better (lower variability) than the precision of TN-K.

Results from the laboratory experiment indicated that the negative bias in TN-A increased as sediment concentration increased but to different degrees for each of the reference materials. Negative bias was smallest for samples prepared with Montana soil and increased for samples prepared with Buffalo River sediment. The potential for reagent limitation in the alkaline-persulfate method was tested by analyzing digests prepared with half the volume of aqueous, nutrient-fortified matrix and twice the volume of persulfate reagent. Nitrogen recovery improved using the enhanced method but was still negatively biased. Supplemental analysis of digest residues provided an almost complete accounting for the missing nitrogen presumed from the negative bias. Therefore, the laboratory experiment established that TN-A has a negative bias, primarily because some PN is refractory (resistant to alkalinepersulfate digestion), leading to sequestration of that nitrogen.

Observed TKN values of the laboratory experiment were positively biased for the Montana soil samples but exhibited both positive and negative biases over a range of Buffalo River sediment concentrations. The positive biases are likely the result of some nitrate being reduced to ammonia and double counted in the calculation of TN-K. This mechanism is well documented during Kjeldahl digestion of soil and plant extracts. The negative TKN biases in Buffalo River sediments were associated with higher concentrations of sediment and may be the result of sequestration of particulate nitrogen within that sediment. In regard to TN-K, the laboratory experiment showed that:

- The Kjeldahl method is substantially less precise (more variable) than the alkaline-persulfate method.
- For sediment-water mixtures typical of conditions in environmental streams, the Kjeldahl method could have a positive bias that may be a result of $\mathrm{NO}_{\mathrm{X}}$ reduction to ammonia or a negative bias as a result of particulate nitrogen that is refractory (resistant to digestion).

Though the variability in TN-A and TN-K biases was considerably greater in the synoptic-field data than that seen in the laboratory experiment, similar trends in those biases were seen in both studies. TN-A bias was evaluated for 905 samples at 77 sites, encompassing total-nitrogen concentrations from 0.15 to about $26 \mathrm{mg} / \mathrm{L}$ and suspended-sediment concentrations from 1 to $14,700 \mathrm{mg} / \mathrm{L}$. Negative TN-A bias was present across the entire range of suspended-sediment concentrations, with relative bias being nearly as great at sediment concentrations below $10 \mathrm{mg} / \mathrm{L}$ as was observed at sediment concentrations up to $750 \mathrm{mg} / \mathrm{L}$. This lends support to the idea that the mechanism of TN-A bias is primarily the sequestration of some portion of PN that prevents complete digestion of nitrogen in the sample. The field study demonstrated that TN-A bias was present in samples with very low suspendedsediment concentrations. At sediment concentrations above $750 \mathrm{mg} / \mathrm{L}$, the negative TN-A bias became more likely and larger, suggesting a secondary mechanism of bias, such as reagent limitation, may be contributing to the negative TN-A bias. Spatial analyses of relative bias in TN-A showed no strong relations to geographical location, topsoil composition, land use, or ecoregion.

The synoptic-field study data indicated both positive and negative biases in TN by the Kjeldahl method. In general, these biases were positive and may have been the result of the reduction of $\mathrm{NO}_{\mathrm{X}}$ during the digestion process.

Four major conclusions were drawn from the synopticfield study:

- The observations from the field are supported by findings of the laboratory experiment.

- Negative bias in TN-A results could occur regardless of suspended-sediment concentration, but it becomes more likely and larger at suspended-sediment concentrations greater than $750 \mathrm{mg} / \mathrm{L}$.

- Relative bias in TN-A does not appear to be explained by variations in the characteristics selected to describe the upstream drainage basin.

- Though variable, positive bias in TN-K could occur in the presence of elevated $\mathrm{NO}_{\mathrm{x}}$.

Understanding the degree of precision and bias in the various total-nitrogen methods is important, but assessing the impacts of that precision and bias on existing and future sampling programs is equally important, especially in the context of a change in analytical method. The anticipated precision and bias of each method need to be considered to avoid creating a new data-quality issue (such as reduced precision) in the 
process of solving another (such as bias). The potential addition of PN analyses to nutrient monitoring programs may incur additional sample-processing time in the field and additional analytical costs. All of the methods have some limitations that affect the quality of the total-nitrogen data and the interpretations that can be drawn from them. Whereas the presence of bias may have an adverse impact on one monitoring program, a discontinuity in the historical record resulting from a methodological change may be considered more adverse to another program. These considerations extend to potential interpretations made by any data user.

This report evaluated three alternative approaches for assessing total nitrogen in surface water: (1) the replacement of TN-A with TN-C; (2) the replacement of TN-K with TN-C; and (3) the addition of TN-C to existing TN-A or TN-K analyses. The primary benefit of replacing TN-A with TN-C is the expected removal of bias from subsequent data, but this approach would also introduce discontinuity in historical records. The concept of replacing TN-K with TN-C would typically apply to a different set of monitoring programs than those currently using TN-A. The primary benefit of this approach is the removal of positive bias in TN-K in the presence of $\mathrm{NO}_{\mathrm{x}}$. However, in addition to the issues that may arise from a discontinuity in the data record, this approach may not be applicable to regulatory programs that require the use of TN-K for stream assessment. If TN-C data were available alongside TN-A or TN-K, biases exceeding a predetermined threshold would be removed while preserving, for the most part, historical data continuity and allowing for a transitional assessment of the impact resulting from bias or a method change. This approach, however, would perpetuate the production of biased TN data, places the greatest strain on field operations, and increases analytical costs more than the other alternatives. The variation in these impacts on different sampling programs will challenge USGS scientists trying to establish uniform standards for total nitrogen sample collection and analytical determinations.

\section{Acknowledgments}

This report is the compilation of several comparative analyses that began with a 10-sample dataset originating from the USGS Georgia Water Science Center and grew to extensive laboratory and field-sampling efforts. The authors wish to thank the following USGS personnel: Terry Schertz, Art Horowitz, and Colleen Rostad for their input on the design of the laboratory experiment and synoptic-field study; Mark Woodworth and the NWQL Nutrients Unit for technical assistance with laboratory experiments; field-sampling crews for collection of additional samples associated with the synopticfield tests; Naomi Nakagaki for ancillary data compilation; and Joel Blomquist, Jeff Martin, Nancy Simon, and Lori Sprague for helpful technical reviews.

\section{References Cited}

American Public Health Association, 2012, Standard methods for the examination of water and wastewater ( $22 \mathrm{~d}$ ed.): Washington, D.C., American Public Health Association, p. 4-130-4-135.

Berman, Tom, and Bronk, D.A., 2003, Dissolved organic nitrogen: A dynamic participant in aquatic ecosystems: Aquatic Microbial Ecology, v. 31, p. 279-305.

Cabrera, M.L., and Beare, M.H., 1993, Alkaline persulfate oxidation for determining total nitrogen in microbial biomass extracts: Soil Science Society of America Journal, v. 57, no. 4, p. 1007-1012.

Capel, P.D., and Larson, S.J., 1996, Evaluation of selected information on splitting devices for water samples: U.S. Geological Survey Water-Resources Investigations Report 95-4141, 110 p., accessed March 2012, at http://pubs.usgs. gov/wri/1995/4141/report.pdf.

Childress, C.J.O., Foreman, W.T., Connor, B.F., and Maloney, T.J., 1999, New reporting procedures based on long-term method detection levels and some considerations for interpretations of water-quality data provided by the U.S. Geological Survey National Water Quality Laboratory: U.S. Geological Survey Open-File Report 99-193, 19 p.

D’Elia, C.F., Steudler, P.A., and Corwin, Nathaniel, 1977, Determination of total nitrogen in aqueous samples using persulfate digestion: Limnology and Oceanography, v. 22, p. 760-764.

Fenneman, N.M., and Johnson, D.W., 1946, Physical divisions of the United States: Washington, D.C., U.S. Geological Survey Map, scale 1:7,000,000.

Fishman, M.J., ed., 1993, Methods of analysis by the U.S. Geological Survey National Water Quality LaboratoryDetermination of inorganic and organic constituents in water and fluvial sediments: U.S. Geological Survey OpenFile Report 93-125, 217 p.

Fry, J.A., Xian, G., Jin, S., Dewitz, J.A., Homer, C.G., Yang, L., Barnes, C.A., Herold, N.D., and Wickham, J.D., 2011, Completion of the 2006 National Land Cover Database for the conterminous United States: Photogrammetric Engineering and Remote Sensing, v. 77, p. 858-864.

Helsel, D.R., and Hirsch, R.M., 2002, Statistical methods in water resources: U.S. Geological Survey Techniques of Water-Resources Investigations, book 4, chap. A3, 510 p.

Hosomi, Masaaki, and Sudo, Ryuichi, 1986, Simultaneous determination of total nitrogen and total phosphorus in freshwater samples using persulfate digestion: International Journal of Environmental Studies, v. 27, p. 267-275. 
Maie, Nagamitsu, Parish, K. J., Watanabe, Akira, Knicker, Heike, Benner, Ronald, Abe, Tomonori, Kaiser, Karl, and Jaffé, Rudolph, 2006, Chemical characteristics of dissolved organic nitrogen in an oligotrophic subtropical coastal ecosystem: Geochimica et Cosmochimica Acta, v. 70, p. 4491-4506.

Mueller, D.K., and Titus, C.J., 2005, Quality of nutrient data from streams and ground water sampled during water years 1992-2001: U.S. Geological Survey Scientific Investigations Report 2005-5106, 27 p., accessed July 2011 at http:// pubs.usgs.gov/sir/2005/5106/pdf/sir2005-5106.pdf.

Nakagaki, Naomi, Hitt, K.J., Price, C.V., and Falcone, James, in press, Methods to characterize environmental settings of stream and ground-water sampling sites for national water-quality assessment: U.S. Geological Survey Scientific Investigations Report 2012-5194.

Natural Resources Conservation Service, 2010, Soil Survey Geographic (SSURGO) Database: United States Department of Agriculture digital data, accessed October 18, 2010, at $h$ ttp://soildatamart.nrcs.usda.gov.

Omernik, J.M., 1987, Ecoregions of the conterminous United States: Annals of the Association of American Geographers, v. 77 , p. $118-125$.

Pace, G.M., MacKown, C.T., and Volk, R.J., 1982, Minimizing nitrate reduction during Kjeldahl digestion of plant tissue extracts and stem exudates: Plant Physiology, v. 69, no. 1, p. 32-36.

Patton, C.J., and Kryskalla, J.R., 2003, Methods of analysis by the U.S. Geological Survey National Water Quality Laboratory-Evaluation of alkaline persulfate digestion as an alternative to Kjeldahl digestion for determination of total and dissolved nitrogen and phosphorus in water: U.S. Geological Survey Water-Resources Investigations Report 03-4174, 33 p., accessed July 2011, at http://nwql.usgs.gov/ Public/pubs/WRIR03-4174/WRIR03-4174.pdf.

Patton, C.J., and Truitt, E.P., 2000, Methods of analysis by the U.S. Geological Survey National Water Quality Laboratory-Determination of ammonium plus organic nitrogen by a Kjeldahl digestion method and an automated photometric finish that includes digest cleanup by gas diffusion: U.S. Geological Survey Open-File Report 00-170, 31 p., accessed July 2011, at http://nwql.usgs.gov/Public/pubs/ OFR00-170.pdf.

Pehlivanoglu-Mantas, Elif, and Sedlak, D.L., 2006, Wastewater-derived dissolved organic nitrogen: analytical methods, characterization, and effects - a review: Critical Reviews in Environmental Science and Technology, v. 36, p. 261-285.
Simon, N.S., and Kennedy, M.M., 1987, The distribution of nitrogen species and adsorption of ammonium in sediments from the tidal Potomac River and estuary: Estuarine, Coastal and Shelf Science, v. 25, p. 11-26.

U.S. Geological Survey, variously dated, National field manual for the collection of water-quality data: U.S. Geological Survey Techniques of Water-Resources Investigations, book 9, chaps. A1-A9, accessed February 2011, at http:// pubs.water.usgs.gov/twri9A.

U.S. Geological Survey, 2009, Potential bias in alkaline persulfate analysis of total nitrogen in whole water samples and recommendations for quantifying bias in whole water samples: U.S. Geological Survey Office of Water Quality Technical Memorandum 2009.03, accessed November 2012, at http://water.usgs.gov/admin/memo/QW/qw09.03. html .

Vandenbruwane, Jeroen, De Neve, Stefaan, Qualls, R.G., Salomez, Joost, and Hofman, Georges, 2007, Optimization of dissolved organic nitrogen (DON) measurements in aqueous samples with high inorganic nitrogen concentrations: Science of the Total Environment, v. 386, p. 103-113.

Wells, D.V., and Ortt, R.A., Jr., 2011, Deep Creek Lake sediment study — Physical and chemical characteristics of lake sediments: Annapolis, Maryland, Maryland Geological Survey, Coastal Environmental Geosciences Program, File Report No. 11-05, 46 p., accessed August 2012, at $h t t p: / / w w w . d n r . s t a t e . m d . u s / p u b l i c l a n d s / p d f s / D C L_{-}$ surficialsediments_final.pdf.

Wyland, L.J., and Jackson, L.E., 1994, Eliminating nitrate interference during Kjeldahl digestion of soil extracts for microbial biomass determination: Soil Science Society of America Journal, v. 58, p. 357-360.

Zimmerman, C.F., Keefe, C.W., and Bashe, Jerry, 1997, Method 440.0-Determination of carbon and nitrogen in sediments and particulates of estuarine/coastal waters using elemental analysis, revision 1.4: Cincinnati, Ohio, U.S. Environmental Protection Agency, Office of Research and Development, 10 p., accessed July 2011, at http://www.epa. gov/microbes/documents/m440_0.pdf. 
Appendixes A1-A5 
Table A1. Results of the laboratory experiment for bias of total nitrogen in prepared water-sediment mixtures analyzed by the alkaline-persulfate method. (link to table in Excel format.)

Table A2. Results of the laboratory experiment for bias of ammonia plus organic nitrogen (total Kjeldahl nitrogen) in prepared water-sediment mixtures analyzed by the Kjeldahl method. (link to table in Excel format.)

Table A3. Unrounded, uncensored replicate data associated with synoptic-field study, 2009-10. (link to table in Excel format.)

Table A4. Sample data associated with synoptic-field study, 2009-10. (link to table in Excel format.)

Table A5. Basin characteristics of selected 2009-10 synoptic-field-study sites where bias in total nitrogen was measured in at least 10 samples by alkaline-persulfate digestion (method TN-A). (link to table in Excel format.)

Publishing support provided by:

Rolla and Lafayette Publishing Service Centers

For more information concerning this publication, contact:

Director, USGS Nebraska Water Science Center

5231 South 19th Street

Lincoln, Nebraska 68512

(402) 328-4100

Or visit the Nebraska Water Science Center Web site at:

http://ne.water.usgs.gov/ 



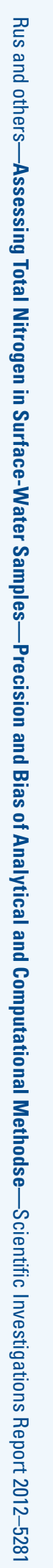

